\title{
Fear, Partisanship and the Spread of COVID-19 in the United
}

\author{
States \\ Robert Kubinec $^{3,} \quad$ Luiz Max Carvalho ${ }^{1} \quad$ Joan Barceló $^{3} \quad$ Cindy Cheng ${ }^{2}$ \\ Luca Messerschmidt ${ }^{2} \quad$ Matthew Sean Cottrell ${ }^{6} \quad$ Derek Duba $^{5}$
}

January 25, 2021

\begin{abstract}
In this paper we argue that the sociopolitical context of the COVID-19 pandemic is as important for understanding the spread of the disease as more conventional factors like social distancing policies and the capacity of the health care system. To do so, we analyze COVID-19 case and test data from the fifty United States during the first seven months on the pandemic. We also devise a Bayesian modeling approach for semi-parametric identification of the infection rate that permits us to undertake more sophisticated covariate adjustment than previous studies, incorporating mediation analysis to better understand why and how sociopolitical, economic and demographic factors affected different channels of transmission. Our results show that partisan identity is a powerful predictor of the spread of the disease by increasing dangerous mobility patterns and reducing people's fear of the pandemic. WORD COUNT: $15,076^{1}$

${ }^{1}$ School of Applied Mathematics, Getúlio Vargas Foundation, Brazil

${ }^{2}$ Hochschule für Politik at the Technical University of Munich (TUM) and the TUM School of Governance,

3 Social Science Division, New York University Abu Dhabi, Abu Dhabi, United Arab Emirates

4 Department of Political Science, University of Southern California

5 School of Politics and Global Studies, Arizona State University

${ }^{6}$ University of California Riverside
\end{abstract} Munich, Germany

\footnotetext{
${ }^{1}$ To reproduce the model and to access the underlying Stan code, please see our Github page. This paper is part of the CoronaNet project collecting data on government responses to the COVID-19 pandemic. For helpful comments we thank participants of the 2020 Polnet/Politics and Computational Social Science Conference. We thank James Bisbee and Rex Douglass for helpful comments. We acknowledge funding from New York University Abu Dhabi and the Technical University of Munich. We thank Tesea Conte, Muhannad AlRamlawi, Shiva Teerdhala, and Luke Burkholder for invaluable research assistance in evaluating state-level COVID-19 policies.
} 
* Correspondence: Robert Kubinec $<$ rmk7@nyu.edu $>$ 
In the United States and in other politically polarized countries, a profound disagreement over how to respond to the COVID-19 pandemic has undermined efforts to control the disease. However, while we have increasing evidence about the relationship between partisan identities and individual beliefs about COVID-19 (Fan, Orhun, and Turjeman 2020; G. Grossman et al. 2020), data about partisan identity, along with other social and economic covariates, are rarely included in efforts to model and predict the spread of the disease in the population (Seth Flaxman 2020; Sharma et al. 2020a; Haug et al. 2020). In this paper we argue that including social, economic and especially political covariates is necessary to obtain a full understanding of the complex patterns of human behavior undergirding the spread of the pandemic.

To expand the scope of modeling contextual factors related to the pandemic, we employ in this paper a Bayesian model that is able to account for biases in COVID-19 testing data yet still enables us to explore in much more detail how the political, social and economic context of COVID-19 influenced the spread of the disease during the first half of 2020. To do so we employ a rich set of adjustment covariates, including voting behavior, public health capacity, socio-economic insecurity, policy responses to the pandemic, and demographic factors. To learn more about how these factors relate to the spread of the disease, we employ mediation analysis to see how much of these associations can be explained by changes in daily state-level polling about concern over COVID-19 and daily Google mobility data. To make the model identifiable, we calibrate our model estimates of the number of infected with state-level serology surveys from the U.S. Center for Disease Control (CDC).

By examining a richer array of covariates and relationships among covariates, we are able to show not only which factors most strongly correlate with rising or falling infections, but also whether these associations are driven by changes in mobility, personal beliefs or other mechanisms. For this reason, even when we cannot make exclusive claims of causal identification, we can still learn about how the associations between covariates and the disease did or did not align with our increasing knowledge about how COVID-19 beliefs translate into human behavior.

We show with this model that even when estimated jointly with other covariates, political partisanship in the United States has a very strong association with the spread of the pandemic. A 1-SD increase in a state's 2016 vote share for Donald Trump is associated with a cumulative increase of $0.5 \%$ to $0.7 \%$ of a state's population infected by SARS-CoV-2 mediated by decreasing people's concern over the pandemic. A 1-SD increase in a state's vote share for U.S. President Donald Trump is also associated with a $0.3 \%$ to $0.5 \%$ increase in a state's infections mediated by increased mobility. We show that offsetting these increases in infections from mobility associated with partisanship would require at least 15 additional days of a state-wide stay-at-home order. 
We also find evidence that political activity on the left is positively associated with the spread of the disease, with states that saw a 1-SD increase in social justice protests following the death of George Floyd witnessing an increase in infections as high as $0.4 \%$ over time. On the other hand, we do not find that the protests reduced people's fears of the disease or changed mobility patterns, suggesting that the spread of the disease happened solely through increased personal contact at the protests and subsequent chains of transmission.

Our model's estimates also shed light on more conventional factors to control the pandemic, especially social distancing policies. We show that the effect of state-level NPIs targeted at the epidemic varies significantly over the time and exhibits trade-offs in terms of reducing mobility. While stay-at-home orders and business restrictions reduced infections by reducing workplace mobility, they also are associated with increased infections in residential places as more people stayed home. These results demonstrate the difficulty in determining the precise effects of policy changes as they are mediated through individual mobility and beliefs about the seriousness of the disease.

\section{Human Behavior and the COVID-19 Pandemic}

A vast and expanding literature documents connections between many political, economic and social factors with human behavior related to the COVID-19 pandemic. While existing studies have shown these associations primarily through surveys and other individual-level analyses, it is difficult to test whether these factors jointly have an effect on COVID-19 infections. The reason for this difficulty is due to how these variables affect human behavior in general equilibrium. For example, non-pharmaceutical interventions (NPIs) like stay-at-home orders have been associated with reduced infections, but stay-at-home orders were also implemented in a rapidly changing environment as public health policies, new suppression practices like masking and the health of the economy varied. People have faced myriad influences on their choices during the pandemic, and even if we have a strong reason to believe that a certain factor should influence their behavior, estimating that effect when many other contravening and contrasting factors were at play is challenging.

At the same time, estimating these general equilibrium effects even within the limitations of available data is very important to learn what factors are associated with the spread of COVID-19 in realistic conditions. For example, some argued that masking would lead to increased infections because it would reduce concern over the risk of infection (Abaluck et al. 2020). Evaluating this hypothesis ultimately requires general equilibrium analysis as it involves competing influences on human behavior. In other words, is the moral hazard of being falsely protected a greater threat than the positive benefits of reducing infections via masking? Being able to sort, rank and understand socio-economic, political and healthcare-related factors behind the disease's spread 
is crucial to better understand why and how COVID-19 overwhelmed countries' disease control systems.

We can summarize the problem of modeling COVID-19 to two main challenges. The first is the challenge of modeling the spread of the disease given the limitations of testing and reported deaths, which could obfuscate the effect of any covariates with data reporting issues. Second, employing observational data requires nuanced comparisons to be made. To learn the effect of a stay-at-home order, for instance, we would want to compare two regions with similar demographic, social and political characteristics as these could be also influencing human behavior, masking the effect of the stay-at-home order. For example, regions with less political partisanship may be more likely to take prudent behaviors to mitigate COVID-19 and also may be more likely to see stay-at-home orders implemented.

In addition, learning about the nature of factors that influenced the pandemic is limited by our knowledge of how different mechanisms contribute to changes in human behavior. We have learned since the outbreak of the pandemic that mobility and personal willingness to follow social distancing and mask-wearing requirements are important intermediate mechanisms that can explain whether these individuals become infected (Bonaccorsi et al. 2020a; Fan, Orhun, and Turjeman 2020; Abaluck et al. 2020; Gao, Rao, Kang, and Kruse 2020). As such, it would be very helpful to know whether covariates of interest influence infections through these known pathways or through some other pathway, especially as some mechanisms like mobility are more amenable to policy intervention than others, such as personal beliefs about the disease.

In this paper, we seek to address these questions by collecting a rich set of important covariates, implementing models to adjust for bias in COVID-19 data and employ mediation analysis to understand the pathways that covariates affect the spread of the pandemic. We believe that doing so contributes to our emerging understanding of the factors that contributed to the spread of the pandemic, especially with respect to factors that we believe tend to be ignored in epidemiological modeling of COVID-19. We argue that political partisanship can be equally as important to the spread of the pandemic as more conventional factors like the implementation of social-distancing measures.

A literature already exists documenting the role of political identity in individual views towards COVID-19, particularly in the United States, but it has largely remained separate from modeling studies of the spread of the pandemic. Political scientists have focused on partisanship due to the way that the pandemic has been politicized along long-lasting cleavages in U.S. politics. The partisan divide in American politics has become a serious concern in political science and the broader community as identities have hardened in a process ongoing since the 1990s or even earlier (Alesina and Rosenthal 1995; K. T. Poole and Rosenthal 2007; K. Poole and Rosenthal 1997; M. Grossman and Hopkins 2016; Iyengar and Westwood 2015). The powerful effect of partisanship on American politics has grown even stronger since the polarizing presidency 
of Donald J. Trump and the hardening of racial identities in the United States (Horowitz, Brown, and Cox 2019). More recently, political scientists have investigated to what extent partisanship has inhibited preventive measures against the COVID-19 pandemic as President Trump has argued against public health policies like face masks. Research has already shown that Republicans are less likely than Democrats to practice public health behaviors like hand washing (Gadarian, Goodman, and Pepinsky 2020), to practice social distancing (Andersen 2020; Alcott et al. 2020; Painter and Qiu 2020), and to comply with policies targeted against COVID-19 (Fan, Orhun, and Turjeman 2020; G. Grossman et al. 2020).

While partisanship in favor of President Trump and the Republican party has received the most attention, other types of political mobilization have also come under scrutiny. Of particular note were the protest movements against police brutality that spread across the United States in the summer and fall of 2020. Existing research suggests the protests have not had an adverse effect on COVID-19 infections (Dave, Friedson, Matsuzawa, Sabia, and Safford 2020), though it is again limited by the observational bias we describe later. As such, it is clear that political motivations on both the left and the right have at times led to reduced compliance with COVID-19 precautions, though it is not clear how severe these factors are, especially when compared to other covariates known to affect COVID-19.

The most important of these, which we also study in this article, are the role of government policies to prevent close personal interaction, which are often classified under the umbrella of non-pharmaceutical interventions (NPIs). Some of the most sophisticated of these studies, which employ state-of-the-art epidemiological models of COVID-19, have examined how country-level differences in the implementation of stay-at-home orders and business closures affected the spread of the pandemic in the critical early period (Seth Flaxman 2020; Sharma et al. 2020a; Haug et al. 2020). While these studies have emphasized the difficult inference issues involved with modeling COVID-19 data, they have largely avoided adjusting NPI estimates with sociopolitical covariates like partisanship, making an implicit assumption that the effect of NPI estimates is independent of these types of human behaviors and identities. For this reason, while these studies help us know much more precisely how NPIs affected the spread of the pandemic through exact measures like the reproduction number, they are more limited in making stronger claims of identification of the NPIs and even through what channels NPIs affect human behavior (Sharma et al. 2020b).

Studies of NPIs and other factors arising in the social sciences, on the other hand, tend to address sociopolitical issues more directly, though they also generally opt for simpler models such as event studies that are easier to estimate with richer covariate sets (Allcott et al. 2020; Dave, Friedson, Matsuzawa, and Sabia 2020). These studies sometimes also employ cell phone mobility data as a proxy for infections with the assumption that reduced mobility will reduce human contact and consequently the spread of COVID-19. 
These studies contain more realism in terms of the factors that could explain COVID-19 at the expense of the more sophisticated methods for modeling the spread of the disease. However, even these studies do not normally include relatively novel factors such as partisanship, and do not generally examine the mediation pathways through which policies and demographics may influence behavior. Furthermore, it is difficult to assess whether the more conventional models employed are able to appropriately address the limitations of COVID-19 data.

Our aim in this paper is to include variables of interest to both the epidemiological and social-scientific literatures, with a particular emphasis on decomposing the channels through which covariates influence human behavior. By doing so, we hope to point out what are in fact the most important factors in the myriad of possible influences on human behavior. One of our central arguments in doing so is that we cannot afford to ignore unconventional factors like partisanship even in models that focus solely on the spread of the disease.

Our argument about the importance of partisanship as a covariate can be expressed with the following two hypotheses:

H1: Higher levels of partisanship as measured by President Trump's 2016 vote share, Trump daily approval ratings and participation in racial justice protests are associated with increased COVID-19 infections even when accounting for government policies and demographic factors.

H2: Increased partisanship in favor of President Trump is associated with increased COVID-19 infections by reducing fears of the severity of the disease and by encouraging risky patterns of mobility.

In the next section, we discuss our statistical method for testing these hypotheses and estimating the effect of NPIs and other factors.

\section{Methods}

Existing models that address these questions generally fall into one of two categories. In the first, models by social scientists investigate how social, economic and political variables affect and are affected by the spread of COVID-19. These models generally attempt to avoid data measurement errors through implementing existing methods, such as time-to-event models and difference-in-difference estimation (Dave, Friedson, Matsuzawa, and Sabia 2020; Courtemanche et al. 2020; Dave, Friedson, Matsuzawa, Sabia, and Safford 2020; Abouk and 
Heydari 2021). In a different vein, a growing literature examines the role of NPIs, or government policies attempting to control the pandemic. These studies tend to use more complicated epidemiological models, known as compartmental models, that are more suited to the complexity of the data, but also tend to have much less sophistication in terms of the range of covariates employed for adjustment (Seth Flaxman 2020; Neil M Ferguson 2020; Haug et al. 2020; Brauner et al. 2020). The more robust modeling of the disease comes at the cost of being able to employ more extensive and more nuanced covariate adjustment, such as the mediation analysis we employ in this paper.

Our approach has a similar aim to the epidemiological models in that it is designed explicitly to model disease dynamics as opposed to re-purposing an existing method which may or may not have valid assumptions for studying pandemics. At the same time, our model can be thought of as a simplification of the compartmental models employed by epidemiologists to study disease, and in particular SARS-CoV2 (Peak et al. 2020; Riou et al. 2020; Robert Verity 2020; Perkins et al. 2020; Jose Lourenco 2020; Ruiyun Li 2020; Neil M Ferguson 2020; Carleton and Meng 2020; Sajadi et al. 2020; Dudel et al. 2020; Tasnim, Hossain, and Mazumder 2020; Seth Flaxman 2020; Brzezinski et al. 2020). These models suppose different classes (compartments) of individuals in the population, denoted $S$ for susceptible, $I$ for infectious, and $R$ for removed (other compartments may be added, such as $E$ for exposed). The model is usually written in the form of a system of ordinary differential equations (ODEs) and assumes a fixed population size, as seems reasonable during a relatively quick epidemic. The number of infected individuals can then be obtained from the solution of the ODE system for the $I$ compartment. These models guide our understanding of the disease and its progression, and have made warnings about the disease's spread that are proving true on a daily basis.

At the same time, these models often struggle to provide straightforward estimates when employing empirical data. This difficulty exists because compartmental models are only identified with a substantial amount of data, such as knowing the exact age mortality distribution of the disease and the time between infection onset and death or recovery. COVID-19 data, unfortunately, has serious flaws that are increasingly wellknown, including limited testing and under-reporting of hospitalizations and deaths (Larremore et al. 2020; Sánchez-Romero et al. 2021). When such data is unavailable, modelers can compensate by simulating plausible random values or using informative prior distributions, but this makes the model estimates tied to the particular set of values used (Grinsztajn et al. 2021). As a result, the challenges in the estimation of compartmental models with empirical data restrict the ability to employ more rigorous forms of covariate adjustment.

By contrast, this paper endeavors to estimate a much simpler quantity than the entire evolution of the outbreak. By so doing, we are able to employ more rigorous empirical modeling of covariates while also 
making fewer assumptions about the nature of the underlying infection process and directly incorporate available empirical estimates of infection. We believe that many researchers and the general public often only want to learn about what has already happened, or the empirical infection rate (also called the attack rate in the epidemiological literature). For a number of time points $t \in T$ since the outbreak's start and states $c \in C$, we aim to identify the following quantity:

$$
f_{t}\left(\frac{I_{c t}}{S_{c t}+R_{c t}}\right)
$$

Assuming a fixed population size, this quantity is simply the marginal rate of infections in the population up to the present. The function $f_{t}$ determines the historical time trend of the rate of infection (which is assumed to be same across countries/regions) in the population up to time $T$, the present. Because the denominator is shifting over time due to disease progression dynamics, this model is only useful for retrospection, i.e., to examine factors that may be influencing the empirical time trend $f_{t}$. As $S_{c t}$ and $R_{c t}$ are exogenous to the model, it cannot predict future prevalence of the disease given that it does not determine these crucial factors.

However, we do not have estimates of the actual infected rate $I_{c t}$, only positive COVID-19 cases $a_{c t}$ and numbers of COVID-19 tests $q_{c t}$ due to the aforementioned measurement issues. Given this limitation, the aim of the model is to backwards infer the infection rate $I_{c t}$ as a latent process given observed test and counts. Modeling the latent process is necessary to avoid bias in using only observed case counts as a proxy for $I_{c t}$. The reason for this is shown in Figure 1 in which a covariate $X_{c t}$, such as a stay-at-home order, is hypothesized to affect the infection rate $I_{c t}$. Unfortunately, increasing infection rates can cause both increasing numbers of observed counts $a_{c t}$ and tests $q_{c t}$. As more people are infected, more tests are likely to be done, which will increase the number of cases independently of the infection rate. As a result, due to the back-door path from the infection rate $I_{c t}$ to case counts $a_{c t}$ via the number of tests $q_{c t}$, it is impossible to infer the effect of $X_{c t}$ on $I_{c t}$ from the observed data alone without modeling the latent infection rate.

Given this overview of the intuition behind our approach, we turn to a more formal definition. Our observed outcomes are the cumulative total of tests and cases reported on a given day $t$ in a given state $c$. We assume that the unobserved state-specific cumulative infection rate $I_{c t}$ can be modeled as a time-varying Beta-distributed random variable with a mean parameter $\mu \in(0,1)$ and shape parameter $\phi>0$. We also assume that the over-time change in the disease can be modeled as a 3-order polynomial time trend that is a function of the number of post-outbreak time periods $T_{O}<T$, where an outbreak begins at the first reported case in a given area. 
Figure 1: Directed Acyclic Graph Showing Confounding of Covariate $X_{c t}$ on Observed Tests $q_{c t}$ and Cases $a_{c t}$ Due to Unobserved Infection Rate $I_{c t}$

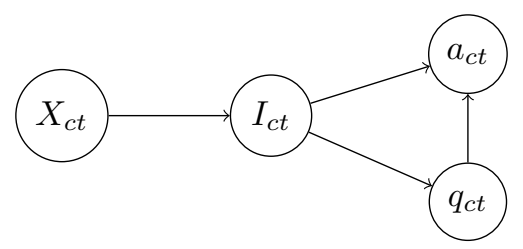

Figure shows the relationship between a covariate $X_{c t}$ representing a policy or social factor influencing the infection rate $I_{c t}$. Because the infection rate $I_{c t}$ influences both the number of reported tests $q_{c t}$ and reported cases $a_{c t}$, any regression of a covariate $X_{c t}$ on the reported data will be biased.

It is important to note that the reason we employ a cubic function is based on theoretical considerations. In our model, the polynomial represents the rate of infection increase in the absence of any other covariates, or equivalently the counterfactual rate of infections. We know from the SIR/SEIR simulations that, in the absence of any countervailing measures, epidemics occur in ever-increasing waves until the herd immunity threshold is reached, although the curve is unlikely to be symmetric as a simpler quadratic function would require. As such, we employ this function because it represents a credible baseline for what the epidemic would do if no other factors impeded its spread. We further allow the polynomial trends to vary by states hierarchically, i.e., the information about the trends is partially pooled across states. The partial pooling is necessary to allow for state-level heterogeneity in the exact progress of the disease, which we note is further modeled by including data on when cases first were reported in each state. We believe this counterfactual infection function is considerably flexible while also motivated by our understanding of how epidemics progress.

We define the conditional distribution of the unobserved infection rate $I_{c t}$ as:

$$
\begin{aligned}
\operatorname{Pr}\left(I_{c t} \mid t=T\right) \sim \operatorname{Beta}(\mu \phi,(1-\mu) \phi) & \\
\mu= & g^{-1}\left(\alpha_{1}+\beta_{O 1} \sum_{c=1}^{C} \sum_{t=1}^{T-14} a_{c t}+\right. \\
& \left.\beta_{I 1} t_{o}+\beta_{I 2} t_{o}^{2}+\beta_{I 3} t_{o}^{3}+\beta_{C} X_{c t}\right)
\end{aligned}
$$

This parameterization of the Beta distribution in terms of $\mu$ and $\phi$ follows from the Beta regression literature (Ferrari and Cribari-Neto 2004) so that we can model the expected value $E\left[I_{c t}\right]$ directly via $\mu$. As such, we use $g^{-1}(\cdot)$, the inverse logit function, to scale the linear model in $\mu$ to the $(0,1)$ interval. For the parameters, $\beta_{O 1} \sum_{c=1}^{C} \sum_{t=1}^{t-14} a_{c t}$ are the sum of observed cases in the country with a 14-day lag, which represents the 
possibility of cross-border spread in infections. The three $\beta_{I i}$ are polynomial coefficients of the number of post-outbreak time periods $t_{o}$.

The parameter vector $\beta_{C}$ represents the effect of independent covariate matrix $X_{c t}$ on the latent infection rate. These are our main variables of interest, and have effects in addition to the polynomial time trends. Finally, the parameter $\phi$ becomes a dispersion parameter governing the variability of latent infection rate.

Because we do not have measures of $I_{c t}$, we need to used the observed data, tests $q_{c t}$ and cases $a_{c t}$, to infer $I_{c t}$. First, we propose that the number of infections will almost certainly increase the number of tests as states try to stop the disease's spread via surveillance. Second, we can assume that a rising infection rate is associated with a higher ratio of positive results (reported cases) conditional on the number of tests, that is, COVID-19 is causing positive test results. We model both of these observed indicators, tests and cases, jointly to simultaneously adjust for the infection rate's influence on both factors. It is this joint modeling that permits us to directly incorporate testing bias. In fact, our model learns about the infection rate from the level of tests rather than trying to discard this information.

To model the number of tests, we assume that each state has an unobserved level of testing capacity, which increases at a non-linear rate during the course of the epidemic. We employ a quadratic function of testing capacity to express the concept of diminishing marginal returns. States were able to ramp up testing once PCR tests were approved by the FDA, but faced constraints due to shortages of supplies, personnel and labs. The cumulative number of observed tests $q_{c t}$ for a given time point $t$ and state $c$ and as a fraction of the states' population, $c_{p}$, then has a binomial distribution:

$$
q_{c t} \sim \operatorname{Binomial}\left(c_{p}, g^{-1}\left(\alpha_{2}+\beta_{b} I_{c t}+\beta_{c q 1} L_{t}+\beta_{c q 2} L_{t}^{2}\right)\right) .
$$

The parameters $\beta_{c q 1}$ and $\beta_{c q 2}$ represent the quadratic increase in testing capacity that varies by state $c$. We similarly allow for partial pooling of these coefficients as testing capacity will show a limited level of variability across states. The parameter $\beta_{b}$ then represents the independent contribution of the level of infections $I_{c t}$ on the total number of requests demanded marginal of testing capacity. The intercept $\alpha_{2}$ indicates how many tests would be performed in a state with an infection rate of zero and at time $t=0$, and as such is likely to be very low.

The binomial model for the number of observed tests $q_{c t}$ provides some information about $I_{c t}$, but not enough for useful estimates. We can learn much more about $I_{c t}$ by also modeling the number of observed cases $a_{c t}$ as another binomial random variable expressed as a proportion of the state population, $c_{p}$ : 


$$
a_{c t} \sim \operatorname{Binomial}\left(c_{p}, g^{-1}\left(\alpha_{3}+\beta_{a} I_{c t}\right)\right)
$$

where $g^{-1}(\cdot)$ is again the inverse logit function, $\alpha_{3}$ is an intercept that indicates how many cases would test positive with an infection rate of zero (approximately equal to the false positive rate of the test), and $\beta_{a}$ is a parameter that determines how hard it is to find the infected people and test them as opposed to people who are not actually infected. The multiplication of this parameter and the infection rate determines the cumulative number of cases, $a_{c t}$, as a proportion of the state population, $c_{p}$.

To summarize the model, infection rates determine how many tests a state is likely to undertake and also the number of positive tests they receive as cases. This simultaneous adjustment helps takes care of misinterpreting the observed data by not taking into account varying testing rates, which has made it hard to generalize findings concerning the disease and also led some policy makers to claim that rising case rates are solely due to increasing numbers of tests. It also allows us to learn the likely location of the infection rate conditional on what we observe in terms of tests and cases.

Because sampling from a model with a hierarchical Beta parameter can be difficult, we simplify the likelihood by combining the beta distribution and the binomial counts into a beta-binomial model for tests:

$$
\begin{aligned}
q_{c t} & \sim \operatorname{Beta-Binomial}\left(c_{p}, \mu_{q} \phi_{q},\left(1-\mu_{q}\right) \phi_{q}\right) \\
\mu_{q} & =g^{-1}\left(\alpha_{2}+\beta_{b} I_{c t}+\beta_{c q 1} L_{t}+\beta_{c q 2} L_{t}^{2}\right)
\end{aligned}
$$

and cases:

$$
\begin{aligned}
a_{c t} & \sim \operatorname{Beta-Binomial}\left(q_{c t}, \mu_{a} \phi_{a},\left(1-\mu_{a}\right) \phi_{a}\right) \\
\mu_{a} & =g^{-1}\left(\alpha_{3}+\beta_{a} I_{c t}\right) .
\end{aligned}
$$

where $I_{c t}$ is now equal to the linear model vector $\mu$ shown in $(3)$ and mapped to $(0,1)$ via the inverse logit function. 


\subsection{Identifiability}

This model contains an unobserved latent process $I_{c t}$, and as such the model as presented is not identified from the data alone without further information. For example, the parameters that control the influence of the infection rate on tests and cases could increase and the latent infection rate could decrease without the probability of the observed data changing.

There two further steps taken to identify this model which we believe represent very limited additional assumptions, especially compared to existing modeling approaches. First, we must require that $I_{c t}$ is a non-decreasing quantity. The number of infected people cannot decrease in an epidemic, but the model as expressed does not require that to be true. ${ }^{2}$ We can eliminate that possibility from the model by imposing an ordered constraint on $I_{c t}$ :

$$
I_{c t}= \begin{cases}I_{c t} & \text { if } t=1 \\ I_{c t-1}+e^{I_{c t}} & \text { if } 1<t<T\end{cases}
$$

This transformation forces $I_{c t}$ to be no less than $I_{c t-1}$. At the same time, we do not need to impose any constraints on the covariates themselves, allowing us to sample those in an unconstrained space before we transform $I_{c t}$.

However, we also need some information about the empirical scale of testing bias to produce identified estimates of $I_{c t}$. We could do so by adding a prior to the model about the plausible range of total infections to reported cases, though we prefer to use information that is more precise. The Centers for Disease Control's serology surveys conducted during the pandemic represent an empirical way of relating $I_{c t}$ to plausible estimates of infections at varying time points. By including this information, we also implicitly account for many of the variables explicitly parameterized in compartmental models such as reporting delays. Because we have an estimate of the number infected at time $t$ that is independent of reported cases and tests, the model will find the parameter estimates that are most likely given the observed differences between the surveys and the reported data.

Though these surveys are opt-in samples, they were adjusted using post-stratification to match population totals, providing a reasonably accurate assessment of the state of infection for a given state (Havers and Krapiunaya 2020). The surveys we employ are listed in Table 1 . In cases where only a portion of the state was sampled, we project the infection rate to the entire state by assuming that the cases/infected ratio

\footnotetext{
${ }^{2}$ Note that we are not assuming that people cannot be re-infected, but rather that people cannot become uninfected after contracting the disease.
} 
(i.e. the observation bias) is constant within the state at that time point.

Table 1: Geographic Serological Surveys from the Centers for Disease Control

\begin{tabular}{llrll}
\hline State & $\%$ Infected & $\mathrm{N}$ & Date Started & Date Ended \\
\hline Connecticut & $4.9 \%$ & 1431 & $2020-04-26$ & $2020-05-03$ \\
Connecticut & $5.2 \%$ & 1800 & $2020-05-21$ & $2020-05-26$ \\
Connecticut & $6.3 \%$ & 1798 & $2020-06-15$ & $2020-06-17$ \\
Connecticut & $5.2 \%$ & 1802 & $2020-07-03$ & $2020-07-06$ \\
Louisiana & $5.8 \%$ & 1184 & $2020-04-01$ & $2020-04-08$ \\
Louisiana & $6.7 \%$ & 770 & $2020-04-22$ & $2020-04-27$ \\
Minnesota & $2.4 \%$ & 860 & $2020-04-30$ & $2020-05-12$ \\
Minnesota & $2.2 \%$ & 1323 & $2020-05-25$ & $2020-06-06$ \\
Minnesota & $4.3 \%$ & 1667 & $2020-06-15$ & $2020-06-27$ \\
Missouri & $2.6 \%$ & 1882 & $2020-04-20$ & $2020-04-26$ \\
Missouri & $2.8 \%$ & 1831 & $2020-05-25$ & $2020-05-30$ \\
Missouri & $0.8 \%$ & 1850 & $2020-06-15$ & $2020-06-20$ \\
Missouri & $1.4 \%$ & 1914 & $2020-07-05$ & $2020-07-09$ \\
New York & $3.69 \%$ & 2482 & $2020-03-23$ & $2020-04-01$ \\
New York & $13.2 \%$ & 1618 & $2020-04-06$ & $2020-04-16$ \\
New York & $16.49 \%$ & 1116 & $2020-04-27$ & $2020-05-06$ \\
New York & $14.44 \%$ & 1581 & $2020-06-15$ & $2020-06-21$ \\
New York & $13.12 \%$ & 1602 & $2020-07-07$ & $2020-07-11$ \\
Pennsylvania & $1.98 \%$ & 824 & $2020-04-13$ & $2020-04-25$ \\
Pennsylvania & $2.48 \%$ & 1743 & $2020-05-26$ & $2020-05-30$ \\
Pennsylvania & $2.64 \%$ & 1694 & $2020-06-14$ & $2020-06-20$ \\
Pennsylvania & $3.55 \%$ & 1751 & $2020-07-06$ & $2020-07-11$ \\
Florida & $0.75 \%$ & 1742 & $2020-04-06$ & $2020-04-10$ \\
Florida & $1.3 \%$ & 1280 & $2020-04-20$ & $2020-04-27$ \\
Florida & $2.12 \%$ & 1790 & $2020-05-19$ & $2020-05-27$ \\
California & $0.96 \%$ & 1224 & $2020-04-23$ & $2020-04-27$ \\
California & $0.94 \%$ & 1539 & $2020-05-19$ & $2020-05-27$ \\
Utah & $2.2 \%$ & 1132 & $2020-04-20$ & $2020-05-03$ \\
Utah & $1.1 \%$ & 1940 & $2020-05-25$ & $2020-06-05$ \\
Utah & $1.5 \%$ & 1976 & $2020-06-15$ & $2020-06-27$ \\
Washington & $0.67 \%$ & 3264 & $2020-03-23$ & $2020-04-01$ \\
Washington & $2.18 \%$ & 1719 & $2020-04-27$ & $2020-05-11$ \\
Washington & $2.19 \%$ & 1803 & $2020-06-15$ & $2020-06-20$ \\
Washington & $1.77 \%$ & 1797 & $2020-07-06$ & $2020-07-07$ \\
\hline & & & &
\end{tabular}

Because we model the infection rate as a cumulative count, it is straightforward to include this information in the model. For a given state $c$ and time point $t$ for which we have survey information, we model the count of infected $S_{c t}^{P}$ as a proportion of the total subjects in each serology survey $S_{c t}^{N}$ with the Binomial distribution:

$$
S_{c t} \sim \operatorname{Binomial}\left(S_{c t}^{N}, g^{-1}\left(I_{c t}\right)\right)
$$


It is important that the serology surveys enter the model in this fashion so that we can model the survey count stochastically. This is necessary to propagate uncertainty in the sample size through to our estimates of $I_{c t}$. This uncertainty matters as well because the serology surveys exhibit random noise and do not always increase over time, as can be seen in Table 1. By modeling the relationship as a probabilistic one, we are making the weaker assumption that the infected rate is probably close to the serology estimate, but the two do not need to be the identical. The combined posterior estimates for $I_{c t}$ will then be weighted with the case and test likelihoods to produce the most credible estimate of $I_{c t}$.

As we show in the supplemental information with simulations, no other identification restrictions are necessary to estimate the model beyond weakly informative priors assigned to parameters.

These are:

$$
\begin{aligned}
\beta_{a} & \sim \operatorname{Normal}(30,10), \\
\beta_{q c i} & \sim \operatorname{Normal}\left(\mu_{q i}, \sigma_{q i}\right), \\
\sigma_{q i} & \sim \operatorname{Exponential}(1), \\
\mu_{q i} & \sim \operatorname{Normal}(0,20), \\
\beta_{C} & \sim \operatorname{Normal}(0,5), \\
\beta_{I i} & \sim \operatorname{Normal}\left(\mu_{I i}, \sigma_{I i}\right), \\
\mu_{I i} & \sim \operatorname{Normal}(0,10), \\
\sigma_{I i} & \sim \operatorname{Exponential}(1), \\
\alpha_{1} & \sim \operatorname{Normal}(0,10), \\
\alpha_{2} & \sim \operatorname{Normal}(0,10), \\
\alpha_{3} & \sim \operatorname{Normal}(0,10)
\end{aligned}
$$

where the normal distribution is parameterized in terms of mean and standard deviation.

The priors to note are the hierarchical regularizing prior put on the varying testing adjustment parameters $\beta_{q c i}$ and varying polynomial trends $\beta_{I i}$ with shared means and standard deviations. This partial pooling permits a reasonable degree of heterogeneity in the parameters while still constraining overall dispersion. Relatively informative priors are put on the hierarchical variance parameters $\sigma_{q i}$ and $\sigma_{I i}$ to suggest that while state heterogeneity does exist, we do still expect the states' estimates to be within a given range. 
We note that a crucial advantage of this framework is providing a way to measured the count of infected adjusting for known biases in the number of tests. By comparing numbers of tests per capita and growth rates in cases across regions, the model is able to backwards infer a likely number of infected individuals in a given area. As such it exploits both within-area and between-area variance to adjust for the biases of imperfect testing. The wide variety of covariates we add to the model, which we describe in the next section, provide the mechanism through which the model can infer test/case relationships even in states which have not had a CDC serology survey.

We also extend this model in order to analyze the mediation of a subset of covariates $X_{c t}^{\prime}$ by adding mediators $M_{c t}$ for mobility and $F_{c t}$ for fear of the disease to the causal diagram, as in Figure 2. Figure 2 has several paths due to the fact that the influence of covariates $X_{c t}$ affects the two mediators differently. Given that beliefs and preferences precede actions, the covariates $X_{c t}^{\prime}$ first influence $I_{c t}$ along the ae and abd path through perceptions of how dangerous the disease is. These beliefs both affect the chance of an individual getting infected and thus $I_{c t}$ directly on the path $a e$, such as by causing an individual to adopt social distancing behaviors, and also on an indirect path $a b d$ by which an increase in a people's fear of the disease reduces mobility as people prefer to stay home.

In addition to pathways through the fear mediator $F_{c t}$, a covariate could influence infections along the pathway through mobility ed without increasing or decreasing fear. This situation could arise if government policies forced people to stay at home against their will and despite their unconcern about the disease. Finally, a covariate could have an unmediated direct effect $g$ on the infection rate. The total effect of a covariate $X_{c t}$ on the spread of the disease is then the sum of all the paths, $a b d+a f+e d+g$. To calculate the indirect effects and direct effects given the use of the inverse logit function $g^{-1}(\cdot)$, we employ the chain rule as in Winship and Mare (1983) to calculate the marginal effect of covariates with respect to different pathways to $I_{c t}$.

Adding the mediators to the model is relatively simple as they do not have link functions and can be included as Normal distributions (i.e., OLS regression) as in Yuan and MacKinnon (2009). It should be noted that there are in fact five mobility covariates as explained in the following section, and so we explicitly model the covariance in mobility via a multivariate Normal distribution with a covariance matrix parameter $\Sigma_{m}$.

To add our mediation covariates $M_{c t}$ and $F_{c t}$, which we describe in more detail in the next section, we multiply the following likelihoods with the joint posterior: 
Figure 2: Directed Acyclic Graph for Latent Infection Rate with Mediators

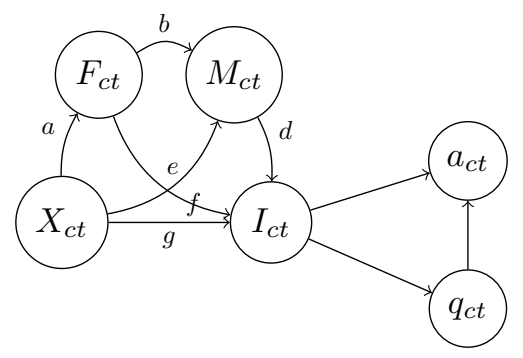

This figure adds mediators $M_{c t}$ (mobility data) and $F_{c t}$ (fear of COVID-19) that mediate the relationship between state-level covariates $X_{c t}^{\prime}$ and the latent infection rate $I_{c t}$. Because beliefs precede actions, $F_{c t}$ is causally prior to $M_{c t}$ and can affect infections both via reducing mobility (path $a b d$ ) and directly apart from mobility (path $a e$ ), such as by encouraging individuals to remain socially distant.

$$
\begin{aligned}
M_{c t} & \sim \operatorname{MVN}\left(\alpha_{m}+\beta_{m} X_{c t}^{\prime}, \Sigma_{m}\right) \\
F_{c t} & \sim N\left(\alpha_{f}+\beta_{f} X_{c t}^{\prime}, \sigma_{f}\right)
\end{aligned}
$$

We also include all of $M_{c t}$ and $F_{c t}$ as linear predictors in (3).

We fit this model using Markov Chain Monte Carlo in the Stan software package (Carpenter et al. 2017). We run the sampler for 1000 iterations with 500 warmup iterations and two chains to test for convergence.

\section{Data}

The only data required to fit the model, in addition to the covariates of interest and serology surveys, are observed cases and tests for COVID-19 by day. In this section, we fit the model to numbers of COVID-19 case counts on US states and territories provided by The New York Times. By doing so, we can use the differences in trajectories across states to help identify the effect of state-level covariates on the infection rate. We supplement these observed case counts with testing data by day from the COVID-19 Tracking Project. As there are discrepancies where the reported number of cases or tests decreases for a given day, we impute these cases and tests through linear interpolation as the number is likely an under/over count of neighboring days. We then take the 7-day rolling average of both series to account for reporting fluctuations and weekly reporting effects.

To analyze the effect of suppression policies, we use data on counts of social distancing policies, restrictions on mass gatherings, restrictions on businesses, mandatory mask orders, restrictions on government services, and 
stay-at-home orders from the CoronaNet Government Response dataset (Cheng et al. 2020). For each type of policy, we include a variable representing the count of policies in that category effective for a particular day. For each update to an existing policy, we code it as +1 if the update increases the scope of the policy or -1 if it decreases the scope of the policy (down to a minimum of 0 ). While this is a simplification of the underlying data, we are still able to capture relative complexity over time without having to make judgments about stringency or other qualitative criteria. We then interact these policy counts with a linear trend to examine time-varying policy effects. We separately include policies designed to increase health resources like personal protective equipment (PPE) and also policies requiring mask use as we do not examine time-varying effects of these covariates. The use of a variety of policy types is important as the adoption of policies is correlated and so including only stay-at-home orders could mask other distinct policies that were implemented at the same time.

The policy data is plotted by state in Figure 3. As can be seen, there is a rise in policies after the pandemic begins in the middle of March, though the number of policies varies across categories. The count of policies is an admittedly imperfect measure though it communicates more information about policy activity than a simple binary coding. Generally speaking, states imposed many more policies designed to increase their access to PPE for health staff than they were willing to take on lockdowns, social distancing, and restrictions on businesses and government services. This difference likely has to do with the increased cost and salience of these policies vis-a-vis relatively less politically difficult options like gathering more masks and face shields for health care workers (Cheng et al. 2020).

To better understand over-time factors that may also affect COVID-19, we include polling data from Civiqs and YouGov at the state level. From Civiqs we include state-level polling averages by day for the percentage of respondents favoring Trump, percentage reporting the economy is "very good," and the percentage reporting that they are "extremely concerned" about the coronavirus. From YouGov we use a poll from May 8th reporting average number of respondents who said they used masks by U.S. state. As this poll does not vary over time, we set the mask prevalence at one-half the minimum value of the poll prior to the WHO's revision of guidance concerning wearing masks on April 3rd, and equal to the poll's values thereafter. As described in the previous section, the poll asking respondents whether they are "extremely concerned" about COVID-19 represents our fear mediator, and is also included as a separate outcome with other covariates as predictors. To better understand the mediating effects of suppression policies, we include Google mobility data ${ }^{3}$ for retail, residential, parks, workplaces, transit and retail establishments. These estimates are by day and aggregated to the state level. They are measured in terms of an index that is initialized with a value of

\footnotetext{
${ }^{3}$ See https://www.google.com/covid19/mobility/
} 


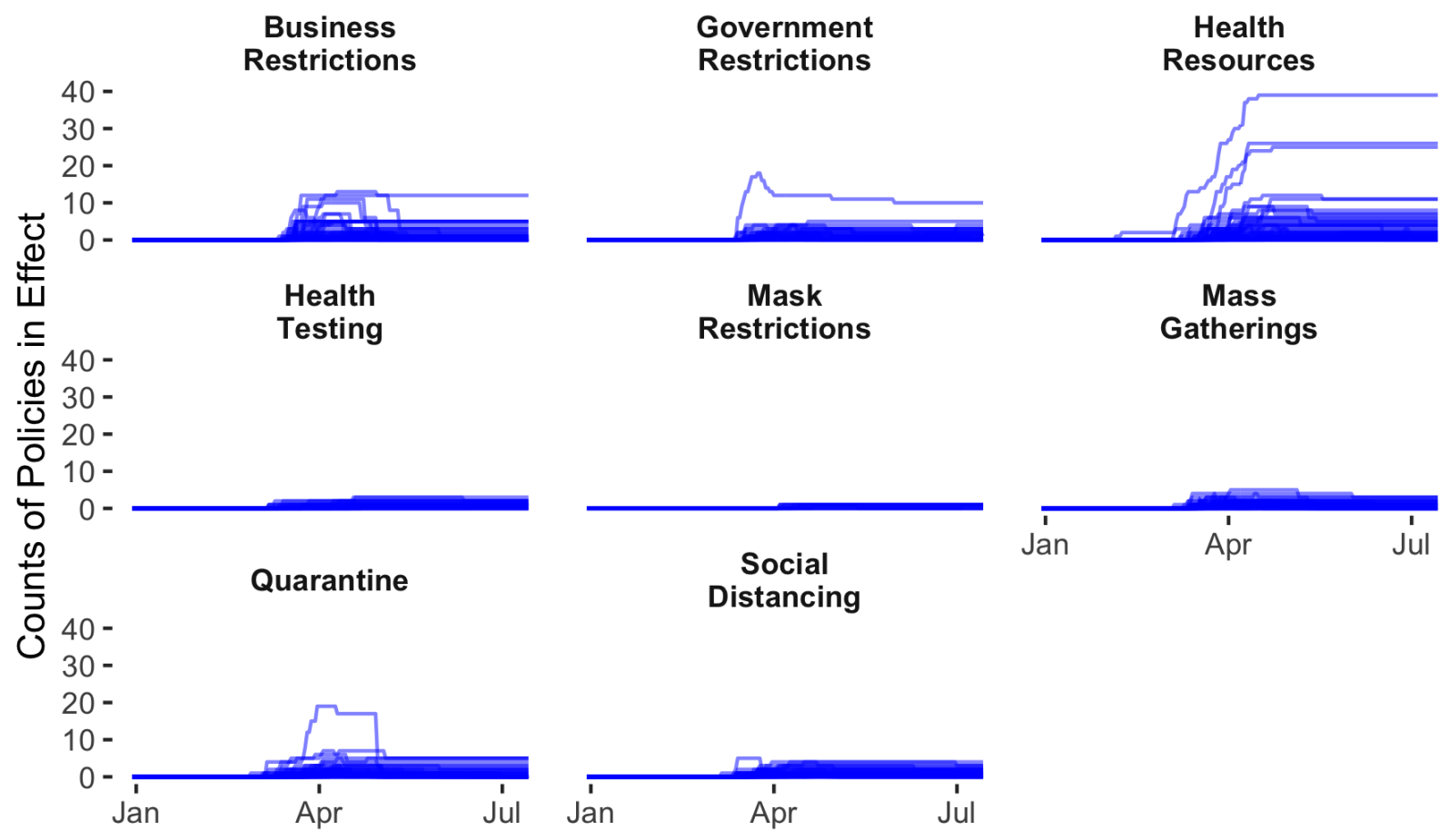

Figure 3: Count of Policies in Effect by Day and by State from the CoronaNet Dataset

100 at the index start on February 15th, 2020. To test for mediation, we include these as predictors of the infection rate, and separately fit a likelihood with each mobility covariate as an outcome and the other covariates as predictors.

We note that it is important to measure mediation for mobility because mobility is hypothesized to affect the spread of COVID-19 (Gao, Rao, Kang, and Kruse 2020). As such, measuring the simultaneous effect on mobility for covariates in our model is important as the covariates could be affecting mobility, which subsequently affects COVID-19 spread. Ignoring this association would result in post-treatment bias that deflates the effect of predictors in the model, though our main interest in including these variables is because this mediation is substantively interesting to decompose.

To measure protest activity, we include a covariate reflecting the proportion of a state's population engaged in social justice protests following the death of George Floyd on May 25, 2020. This data is drawn from publicly available information about the number and size of protests from three online sources: Wikipedia protest data, the Count Love protest web-crawling web site, ${ }^{4}$ and list of protests compiled by Ipsos. ${ }^{5}$ For protests present in only one of the three sources, we used information on both size and location. If a protest

\footnotetext{
${ }^{4}$ https://countlove.org/

${ }^{5}$ See https://www.ipsos.com/en-us/knowledge/society/Protests-in-the-wake-of-George-Floyd-killing-touch-all-50-states
} 
was present in three sources, we averaged reported protest size. If the sources had contradictory information about the type of protest, we had research assistants re-code the protest using secondary sources. For protests for which size was not available, we imputed missing data using random forest algorithms (Stekhoven and Bühlmann 2012).

All time-varying covariates-polling, protests, policies and mobility data-are lagged by 14 days to account for the likely delay in events showing up in reported cases. This 14-day lag comes from the epidemiology literature (Seth Flaxman 2020) and is meant to take into the account the amount of time required for people to be infected, be tested and then have the test results reflected in case counts.

We further add in non-varying state-level data on Donald Trump's vote share for the 2016 election from the MIT Election Lab, a 2019 estimate of state GDP from the Bureau of Economic Analysis, the 2018 percentage of foreign born residents, population under 18 years of age and population density from the U.S. Census Bureau, 2019 state-level average data on air pollution, ${ }^{6}$ cardiovascular deaths per capita, percentage of residents under age 18, number of dedicated health care providers, public health funding, and smoking rates provided by the United Health Foundation ("America's Health Rankings 2019 Report" 2019). All variables are standardized to permit comparability.

We employ state-level data rather than country-level data because our aim is to have a rich adjustment set of covariates. While some of our data is available as well at the country level, crucial covariates such as polling about fears of COVID-19 and the state of the economy are only available at the state level. We believe that obtaining quality estimates of these crucial variables is more important than the statistical power we would obtain from dis-aggregation.

This is particularly true because in general we cannot make claims of causal identification as we can with our claims of statistical identification of the latent infection rate. COVID-19 is not a very likely candidate for meeting any kind of assumption about ignorable selection into treatment; it is a disease that is indirectly caused by human behavior. Our identification strategy primarily relies on including as many relevant adjustment variables as is prudent to isolate factors which are likely to or known to have an effect on COVID-19 spread and could be confounding variables.

In addition, even when we cannot ensure causal identification, we can still learn important aspects of the underlying relationships by partitioning the variance via mediation analysis. Doing so allows us to isolate the part of the association which we have a strong theoretical reason to believe is causally related to the spread of COVID-19, such as via influencing people's fears over the severity of the virus in their area. While

\footnotetext{
${ }^{6}$ Defined as average exposure of the general public to particulate matter of 2.5 microns or less $\left(\mathrm{PM}_{2.5}\right)$ measured in micrograms per cubic meter (3-year estimate).
} 
using observational data necessarily means there are inferential concerns we cannot rule out, we can still learn substantially from variables that we have a strong prior reason to believe are related to the outcome.

\section{Results}

We first examine model performance to see how well the model is able to reproduce the observed data. While this kind of predictive validity is only partially useful given that we are interested in a latent quantity, it is still helpful to know whether the model is able to reproduce the empirical distribution. If the model could not fit the observed data very well, then we might be suspicious about whether we are informing our latent estimate correctly. To do so we estimate the posterior predictive distribution, i.e., we draw from the posterior distribution using the empirical data.

The results of drawing from posterior values for the beta-binomial distribution of cases and tests are shown relative to the original observed values in Figure 4 for five states. The plots show that although there is noise in the predictions (represented by the black shaded region), the model is generally able to capture the empirical values (represented by a red line) with relatively little uncertainty for confirmed cases. However, the relationship is more stochastic for test counts. While the model predicts that tests rise over time, it also allows for the possibility that test counts could have been higher or lower. This difference in predictive performance flows from the model as there are other factors which may impact testing whereas the latent trait is most directly related to confirmed case counts. Given that our intention is in bias adjustment rather than predicting test counts, the noise in the relationship for that part of the model is acceptable. To the extent that a relationship exists between tests and the infection rate, it will still be accounted for in the posterior estimates.

Given this check on the model's fidelity to the data, we can then report the model's estimates of infected counts for the U.S. population as a whole in Figure 5. Panel A in this plot shows the cumulative total both for reported cases (thin black line) and for the model's estimate of total infected (blue line). The interval in this plot, as with all figures presented, are the $5 \%$ and $95 \%$ quantiles of the empirical posterior distribution. As can be seen, the model estimates that there are approximately 3-4 times as many infected people in the United States as reported cases, with the total cumulative number of infected persons reaching 15 million with around 500,000 infected as of mid-July. Early expert estimates are shown as black points in panel A, revealing that even epidemiologists largely under-estimated the spread of the disease in its early stages, largely due to limitations in testing and case reporting.

We compare these estimates with a popular COVID-19 forecaster employing SEIR models from Gu (2020) by 
A

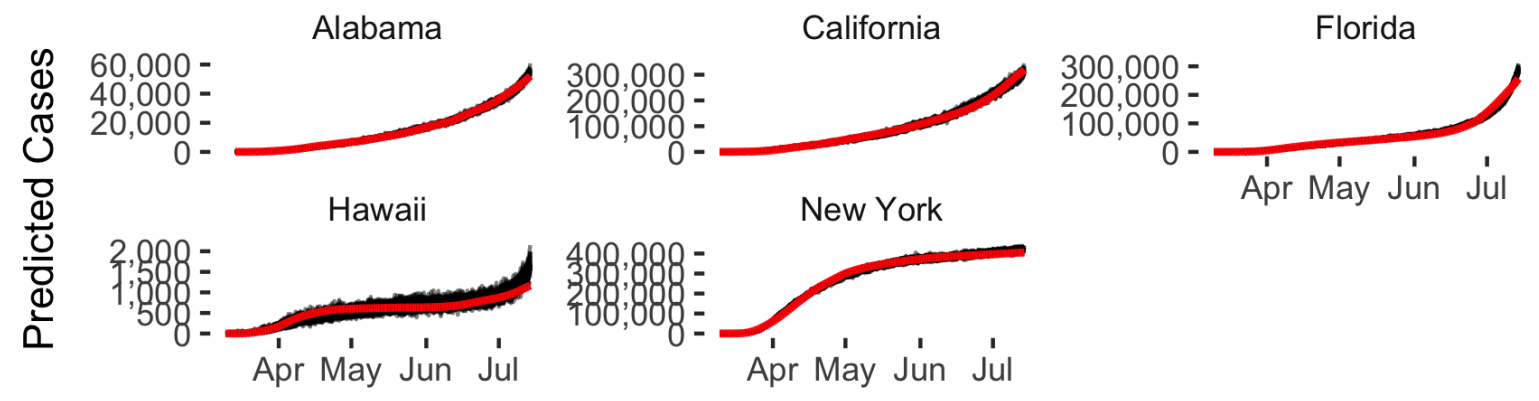

B

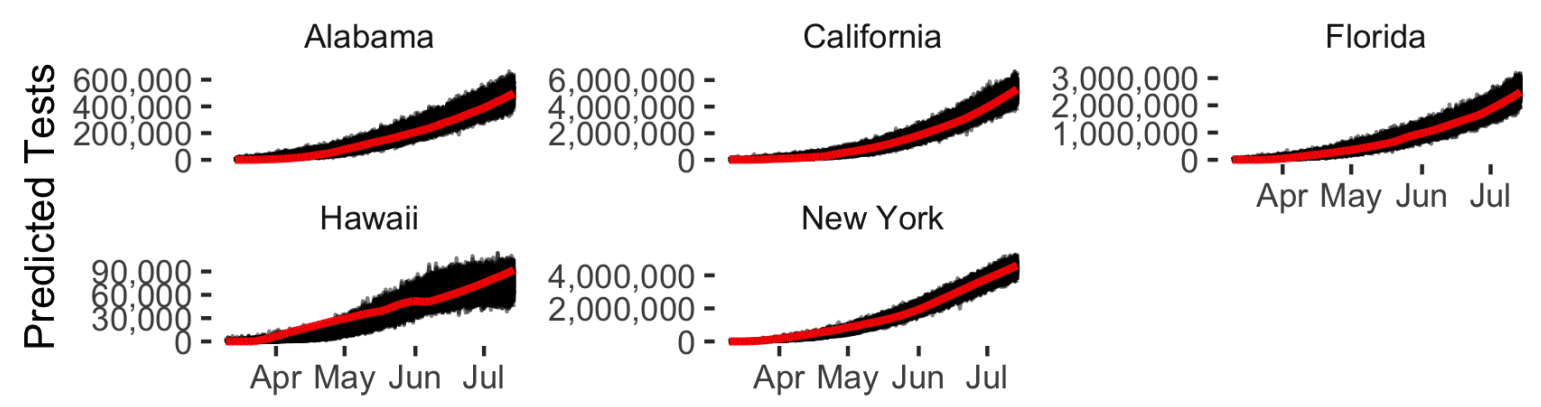

Plot shows posterior predictive values for cases and tests in black and the value from the data in red. The black region represents the $5 \%$ to $95 \%$ quantiles of the empirical predictive posterior distribution.

Figure 4: Predictive Validity of Model Vis-a-vis Observed Cases and Tests 
plotting their estimates as a yellow ribbon on the plot. As can be seen, the trajectories are similar although they diverge slightly at the end of the series in mid-July. This is likely due to the fact that our model is not designed to predict into the future, and so it does not pick up as strongly on the increase in cases in the last week of July. However, on the whole it would seem that our estimate of infected individuals is on the conservative end compared to other approaches-in other words, while we do not know for certain what the true number is, we are unlikely to be under-estimating the total. Again, as our aim here is interpretation and inference rather than forecasting, we believe the conservative nature of our model is a benefit. The estimates of covariate associations we present later in this paper could be slightly too low, but they are unlikely to be too large given what we can model. Furthermore, our intervals are far more precise than other approaches, which is likely because we employ extensive covariate adjustment to better infer human behavior during the course of the pandemic.

Panel B in the plot shows our estimates of infected individuals, excepts that it adjusts the cumulative number with a 19-day lag to account for the approximate time that recovery from COVID-19 requires (deaths are first subtracted). This plot displays an imperfect but useful formulation of the likely number of people infected at any given time point. As of July 14, it would appear that there were approximately 500 thousand infected individuals in the United States, while the number peaked at about four million in late April. ${ }^{7}$

By comparison, Figure 6 shows the cumulative totals of estimated infections by state. Plot A in this figure has the count of infections by state, while plot B shows the percentage of the population infected by state. Both the overall S-shape of the epidemic can be seen along with the substantial heterogeneity in infections, with early infected states like New York and New Jersey still in the top quartile of states with infections even though they successfully reduced the rate of disease spread.

In addition to the estimation of the cumulative count of infected individuals, the model provides further useful information by parameterizing the relationship between the unobserved infection rate and the number of tests conducted in a given state. These individual parameters are shown in Figure 7. The scale of the y axis shows the number of people that a state was able to test relative to each person infected. The plot shows that some states have been able to test far more people than have been infected (New York, New Jersey), while other states like Pennsylvania and Oregon have tested barely twice as many as those who have been infected. The fact that new outbreaks have been seen in Texas and Arizona suggests that this shortfall in testing likely disguised early outbreaks that could have been detected otherwise.

In addition, we know from Figure 7 that because New York and New Jersey are quite high in the test/case

\footnotetext{
${ }^{7}$ While it is interesting to note that the model shows fewer infected people than observed cases for this plot at the end, this difference is merely an artifact of the 19-day lag. It is difficult to put a precise number on the presently infected as states do not always report how many recover from COVID-19.
} 
A

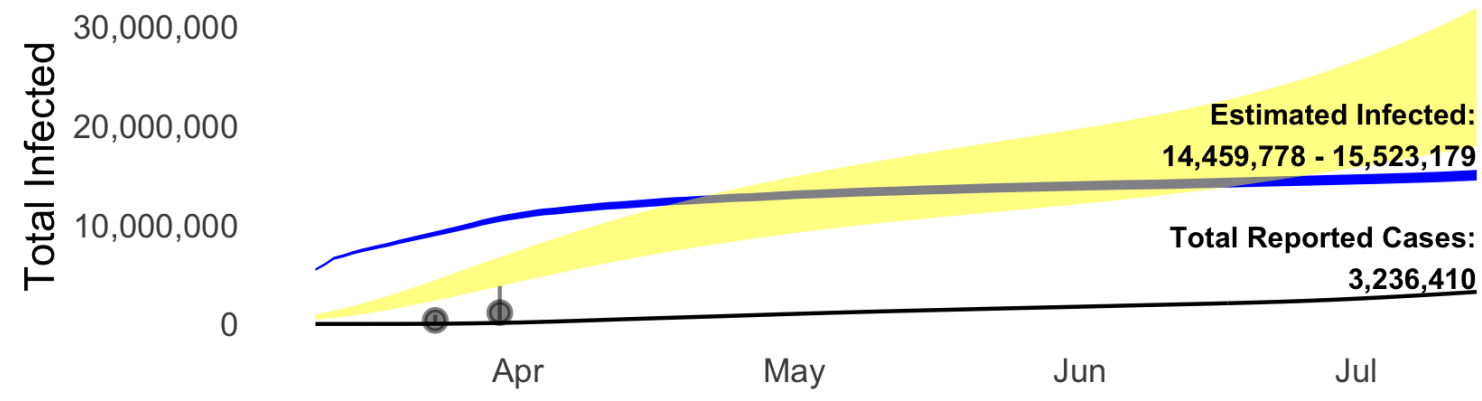

$\mathrm{B}$

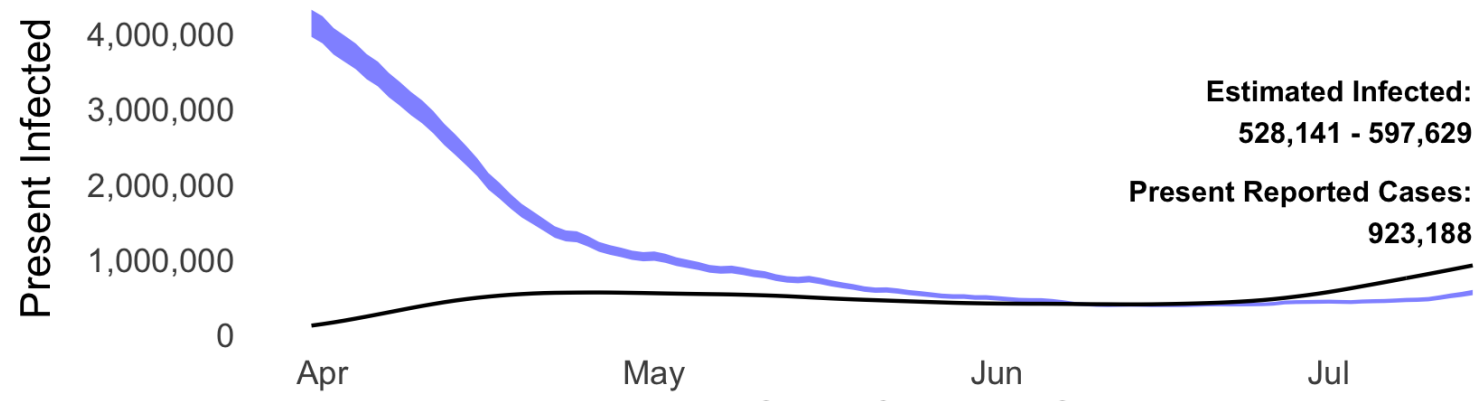

Days Since Outbreak Start

Blue 5\% - 95\% HPD intervals show estimated infected and the black line shows observed cases from he New York Times. These estimates are based on CDC seroprevalence data and a Bayesian model of how cases and tests are influenced by infection rates. Black dots in Panel A show early expert estimates of COVID-19 prevalence in the United States. Yellow ribbon shows 5\% - 95\% predicted cumulative infections from covid19-projections.com hybrid SEIR model.

Figure 5: Total Cumulative and Present COVID-19 Infections in the United States 
A

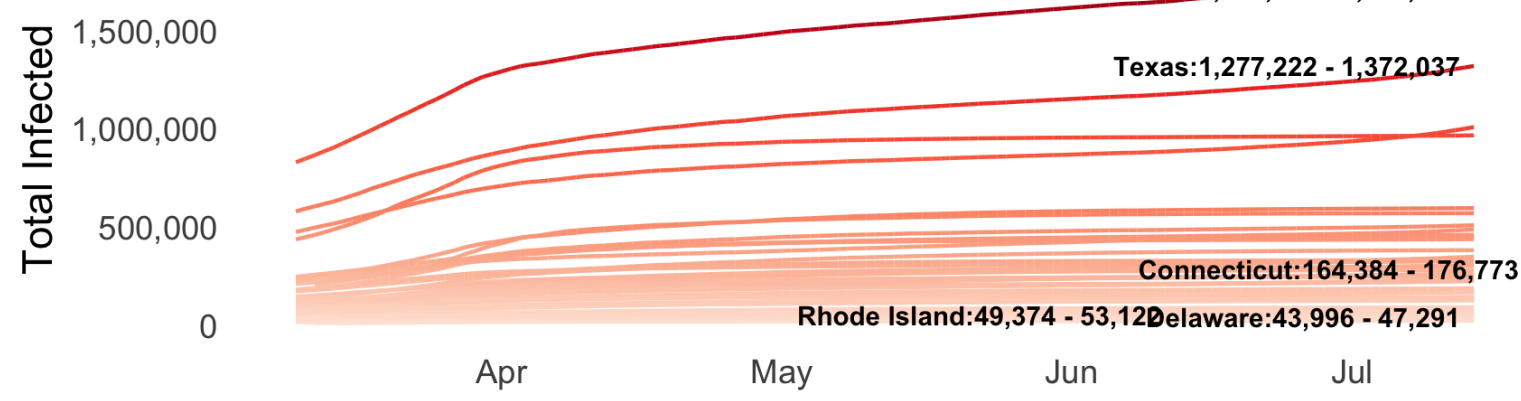

California:1,726,660-1,853,214

B

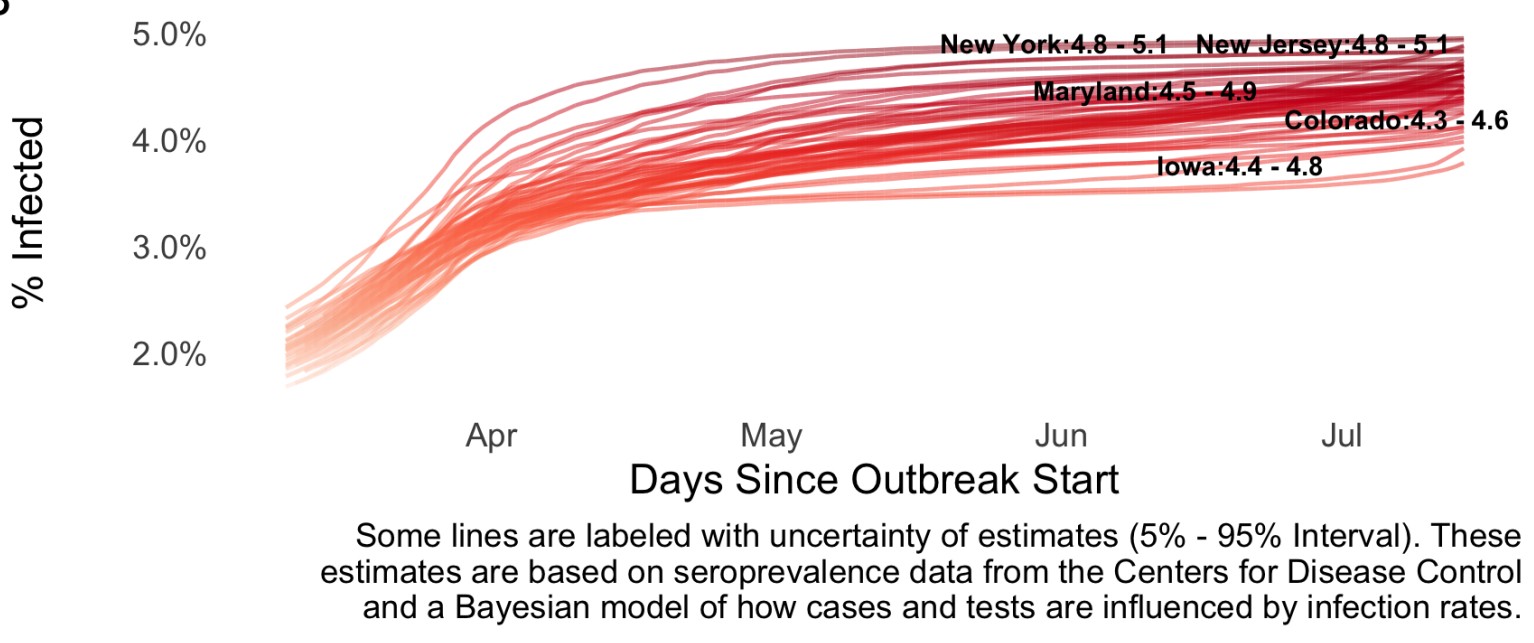

Figure 6: Average Cumulative Count of Infected People by U.S. State as of July 14th 
distribution, having tested around four individuals per infected person, the high infection rates in Figure 6 for these states are not an artifact of more rigorous testing. The model is successfully able to separate the bias of increased testing from the actual level of new infections.
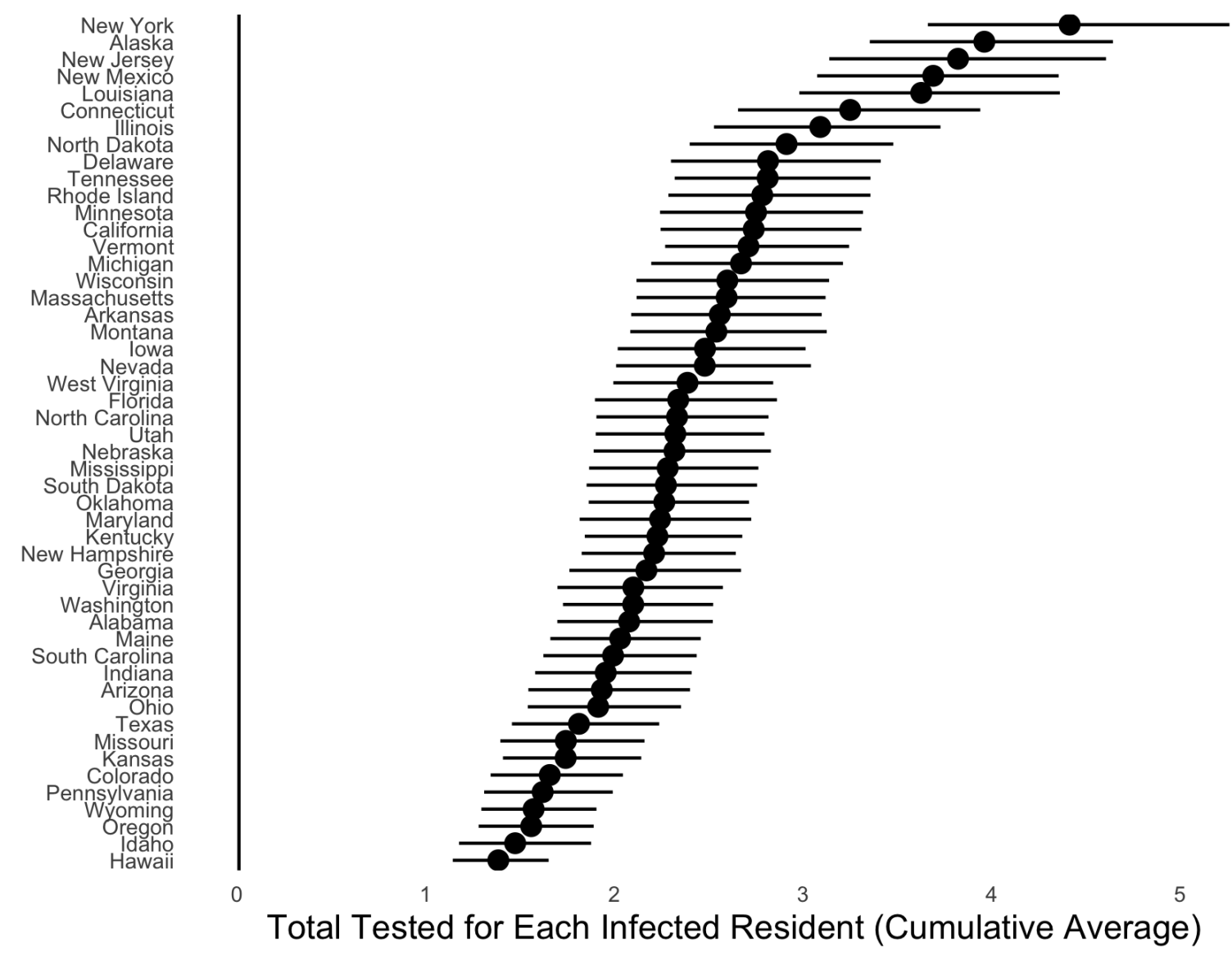

Figure shows the average number of additional people tested in a given state for each person who becomes infected. Estimate is a cumulative average of the last seven days of data.

Figure 7: Measuring States' Testing Rates Relative to Infection Rates

We would note that this information is also helpful to policy makers and others trying to make sense of observed case counts given the limitation in testing thus far. Our estimates help take into account these known biases and adjust them based on differences between states and within states in terms of disease trajectories. We believe this model can be used to help understand disease trends and factors associated with it even in the relatively data-poor environment many countries find themselves in. Unlike SEIR/SIR approaches, we do not employ information about hospitalization and death reporting delays, the infectionage distribution, or initial seeds. While these other outcomes can provide additional information about the progress and severity of the disease, they also considerably complicate inference. 
To calculate the effect of covariates on the infection rate, we report here average cumulative marginal effects by state, i.e., by how much a given covariate increased the proportion infected for a one-unit increase in the covariate over time. We report cumulative marginal effects rather than the sample average marginal effect because the outcome monotonically increases, and so the marginal effect at any one point in time is not as meaningful a statistic. The way to interpret the coefficients presented is how a 1-unit (1-SD) change would affect the infection rate if that increase were sustained for an average state's entire time series (March to July).

We first show the association of mobility types with the infection rate. In Figure 8 we show the marginal effect of a 1-SD increase in different types of Google mobility on the infection rate expressed as a fraction of a state's population. In line with the growing research on cellphone mobility and the epidemic, there are strong positive effects of some types of mobility on the spread of the disease, especially residential, workplace, and retail mobility. Movement in parks, on the other hand, is negatively associated with COVID-19 occurrence. While these results are somewhat surprising given that both residential and workplace mobility are very large, other results confirm with prior suspicions that outdoor activities like attending parks are relatively low-risk for COVID exposure. In fact, increased mobility in parks is associated with reduced infections, probably because it substitutes for more high-risk types of mobility.

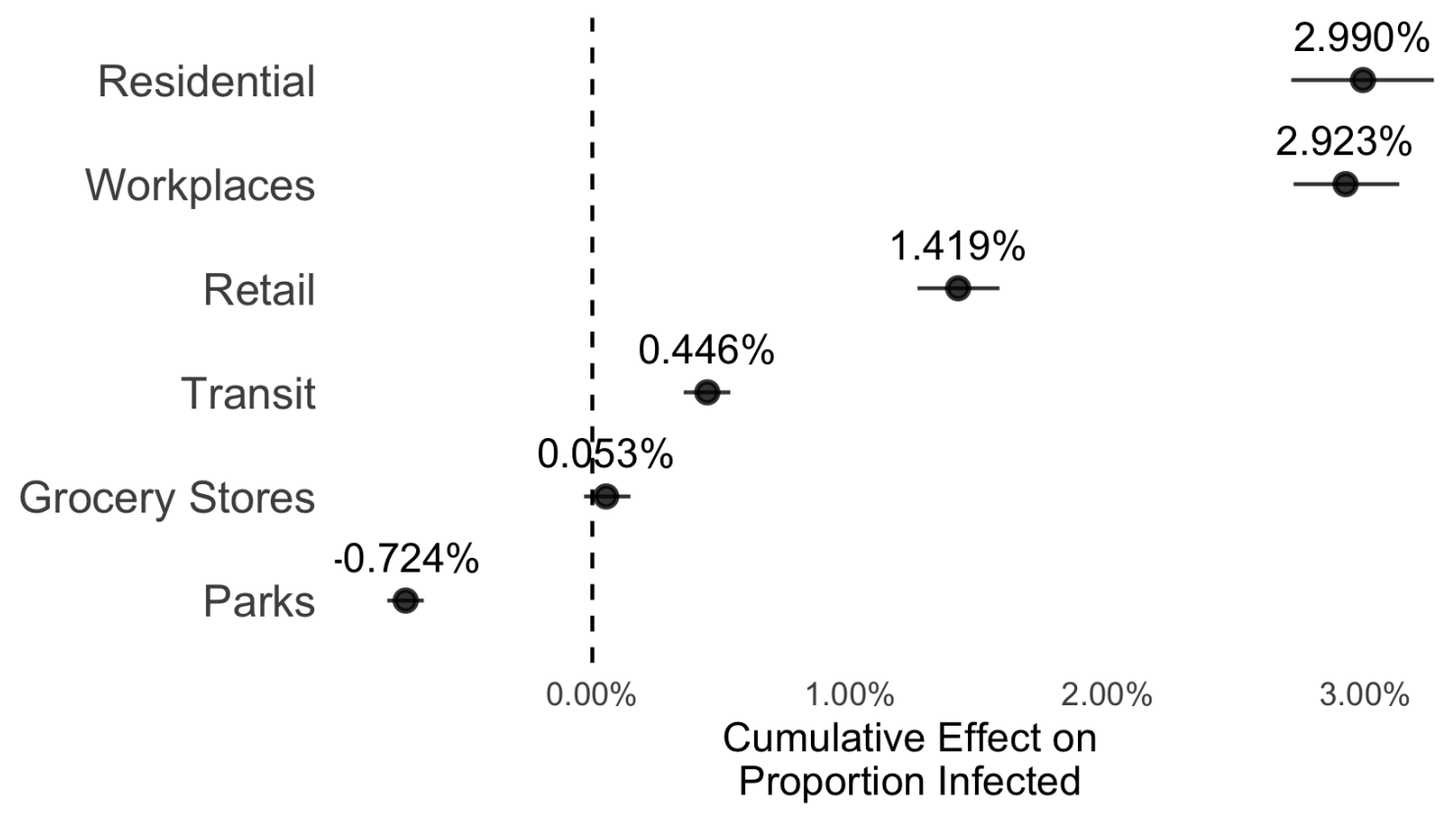

Marginal effects calculated as a 1-standard deviation change in a covariate on the cumulative latent infection rate. $5 \%-95 \%$ high posterior density intervals derived from 1000 Markov Chain Monte Carlo posterior draws.

Figure 8: Effect of Google Mobility Data on COVID-19 Spread 
To interpret these coefficients correctly, it is important to take into account the multivariate normal distribution that was used to model each of these mobility measures as one joint distribution. The residual correlations for the mobility measures model are shown in Figure 9. These correlations are intuitive, with transit positively correlated with other mobility measures except residential (people tend to be at home if they are not in transit). What is quite important is that workplace and residential mobility are strongly inversely correlated at -0.88 ; in other words, people tend to be at home if they are not working and vice versa. As a result, the effect of residential and workplace mobility on COVID-19 is complicated due to this displacement effect. The fact that residential mobility is positively associated with infections once this displacement effect is taken into account accords with the modeling literature that warned that stay-athome orders would paradoxically increase infections in the home as people were kept in close quarters with each other (Neil M Ferguson 2020). We believe these strong correlations provide compelling evidence for employing the multivariate normal distribution in our model so that we do not assume these measures are conditionally independent. At the same time, it does render the interpretation of mediation effects somewhat more complicated as the model is explicitly taking into account that changes in one type of mobility are likely to displace or effect other types of mobility.

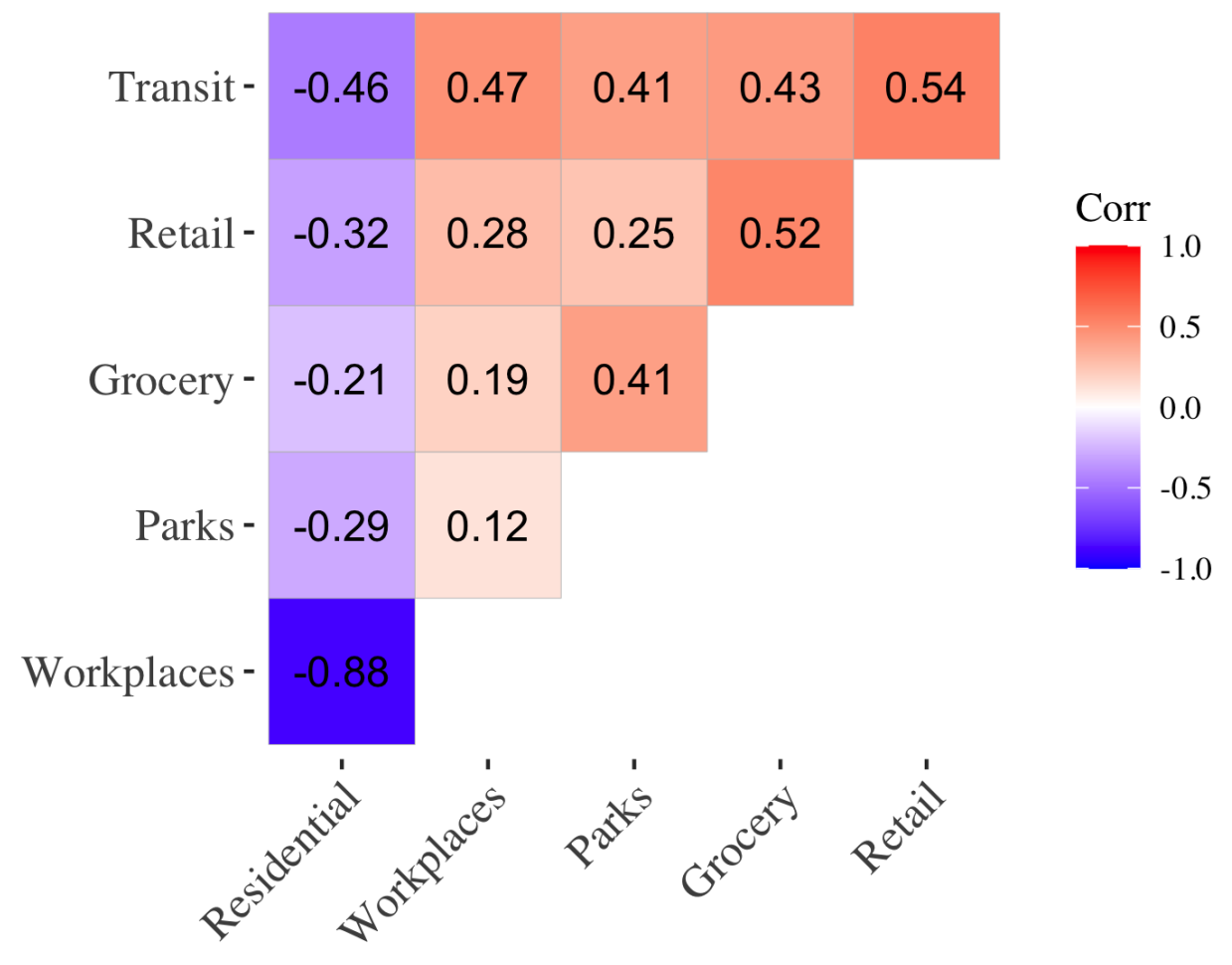

Figure 9: Estimated Correlation of Mobility Measures

We next turn to an analysis of the rest of the covariates used to predict the latent infection rate. Figure 10 
shows the marginal effect of all other covariates in the model on the latent infection rate expressed as average cumulative marginal effects. The estimates are further broken out in terms of mediation. The mobility effect is equivalent to the $e d$ path in Figure 2, i.e., it is the path from the covariates to mobility that does not go through increased fear of COVID-19 measured by daily polls. The fear of COVID-19 pathway, on the other hand, is equivalent to the $a b d+a e$ paths, or the sum of the path from fear through mobility and the path from fear to infections apart from mobility. In other words, a covariate's effect mediated by increased fear of COVID-19 can both immediately impact the outcome by heightening sensitivity to the severity of the pandemic and affect the outcome by reducing an individual's willingness to engage in dangerous types of mobility. The direct effects, which represent the unexplained effect of covariates independent of either concern over COVID-19 or changes in mobility, are then equivalent to the $g$ path in Figure 2, and the total effects are the sum of all paths. The direct and indirect effects are disaggregated in panel A while the total effects are shown in panel B.

The use of mediation analysis shows substantial heterogeneity in the types of associations and whether direct and indirect effects tend to complement or substitute each other. First, it is important to note that the single strongest associations in panel B come from the YouGov mask-wearing poll, the Civiqs concern over coronavirus poll and the economy poll, and the percent of a state's residents that are foreign-born. As these are cumulative average marginal effects, that number reflects what an average state might experience; the effect could well be larger for states with higher infection rates than average. On the other hand, as these effects are cumulative, they reflect a state that experienced a sustained increase in the covariates and so it might overstate the effects somewhat.

In terms of total effects in panel $\mathrm{B}$, the factors that are most strongly associated with reducing infections include concern over COVID-19, wearing masks, concern over the state of the economy, higher per capita income and higher population density. Conversely, a higher percentage of foreign-born, more social justice protests, PPE policies and greater concentrations of $P M_{2.5}$ are associated with more infections. Some of these associations would correspond to what is known about the virus, such as mask-wearing reducing infections while large gatherings like protests increasing infections.

Mediation analysis is helpful at understanding what may be driving these associations. We can learn more about the meaning of the results when we can identify effects through pathways which we have a theoretical reason to believe matter for fighting the epidemic: individual concern over COVID-19 and individual mobility. For example, while the association with percent foreign-born is quite strong, we know that the bulk of this association arose through an unspecified mechanism. Indirectly, the percentage foreign born is associated with reduced COVID-19 spread via the fear pathway. Instead, it is most likely the case that the percentage 
A

Direct and Indirect Effects

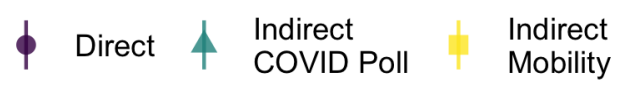

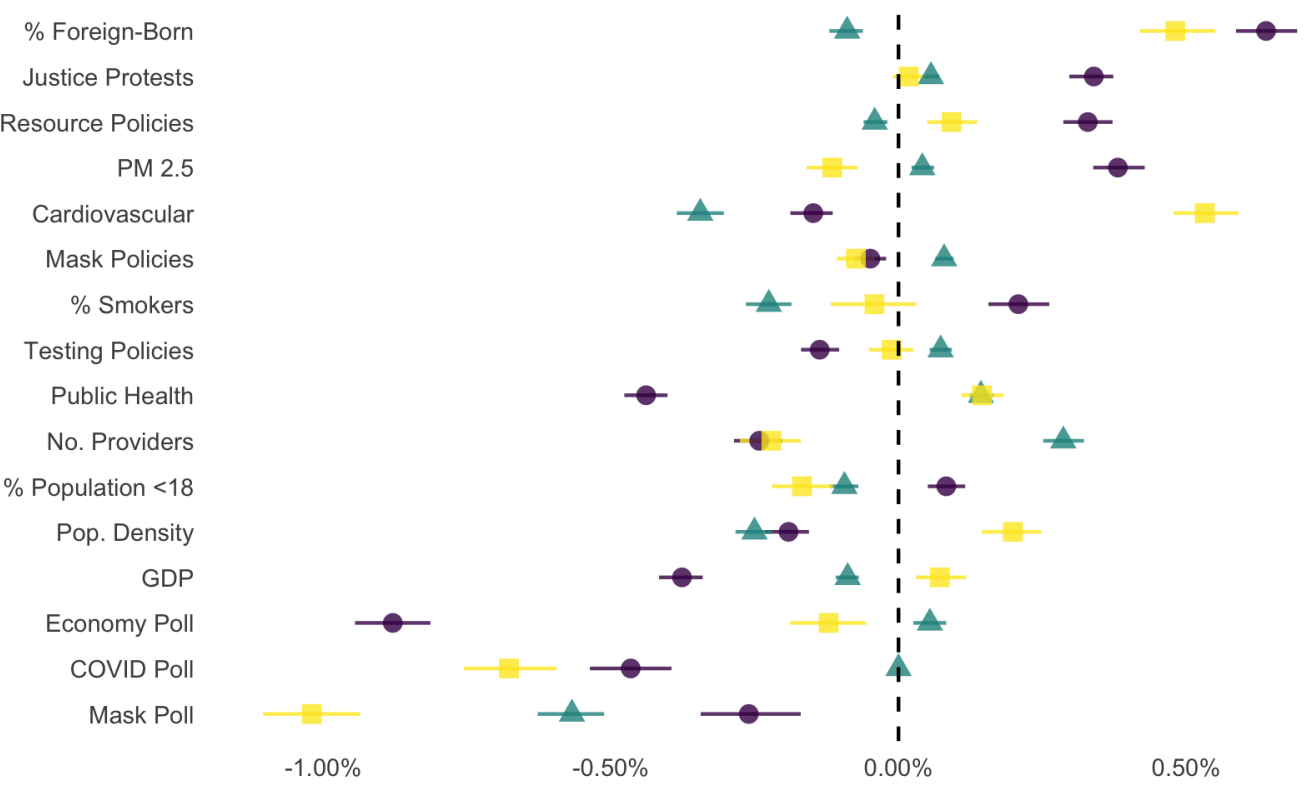

B

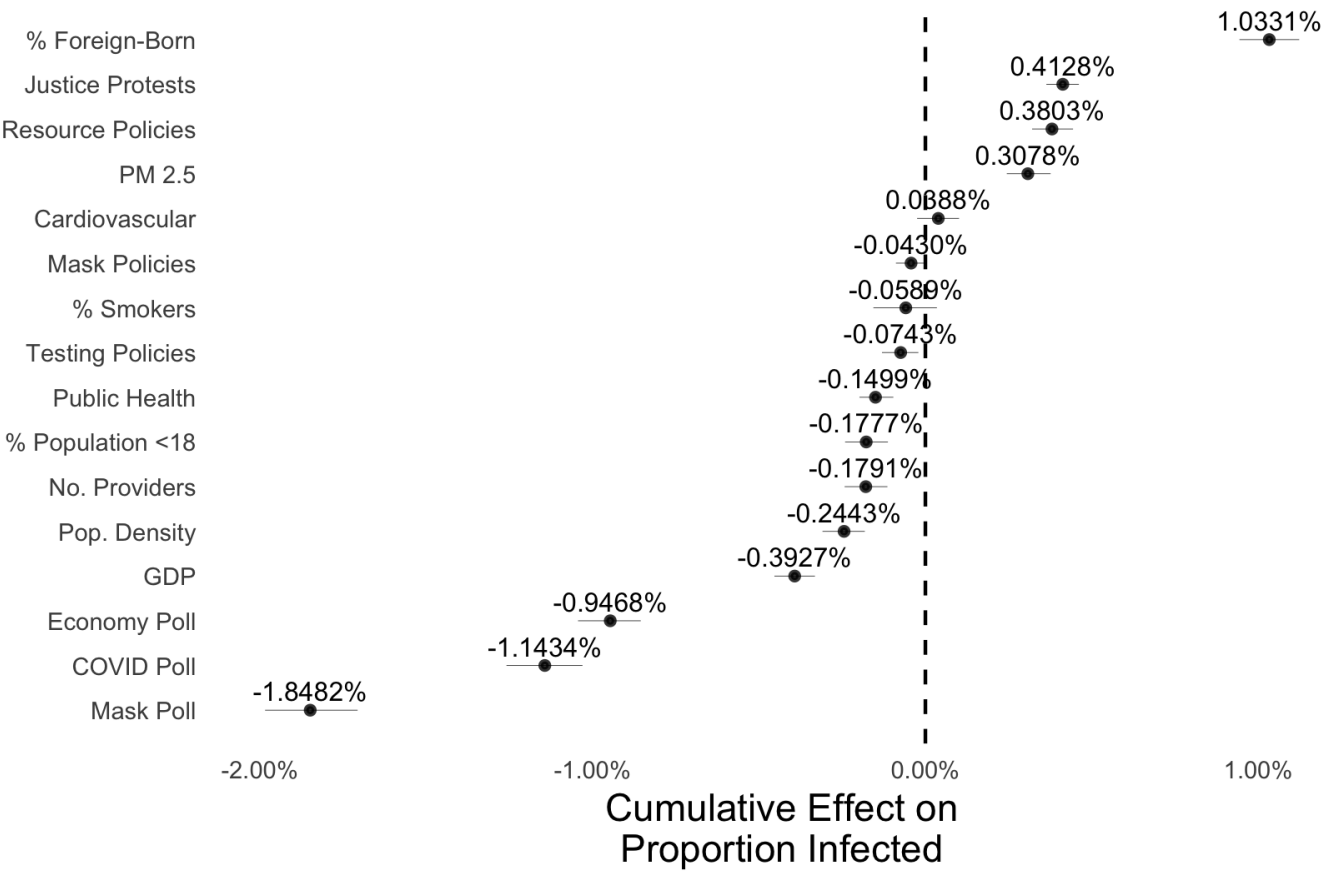

Marginal effects calculated as a 1-standard deviation change in a covariate on the latent infection rate. $5 \%-95 \%$ high posterior density intervals derived from 100 Markov Chain Monte Carlo posterior draws.

Figure 10: Marginal Effects of Covariates on Latent Infection Rates for U.S. States 
of foreign born is a proxy for international travel which led to initial outbreaks. This proxy was the reason the covariate was introduced into the model as it is difficult to otherwise capture travel patterns that may have introduced the virus earlier.

In contrast to other research, we find that social justice protests are positively associated with COVID-19 spread, though the effect is of medium size. Furthermore, as we report cumulative marginal effects, it is unlikely that states experienced protests every day in the sample, suggesting that the reported effect is more of an upper bound for what most states experienced. We do not find much evidence, as Dave, Friedson, Matsuzawa, Sabia, and Safford (2020) suggest, that the positive effect of the protests was offset by reduced mobility by non-protesters as the indirect effects are almost zero. On the other hand, the effect of the protests could have been much worse if it had decreased people's fears of the virus or induced risky travel patterns. In this case, the direct effect is relatively easy to surmise: close contact through the protests which spread infections. It is important to note as well that this effect exists even controlling for both Trump vote share and Trump approval rating, so it is not simply a proxy for state-level partisanship.

There are other interesting associations in Figure 8. States with more people with cardiovascular issues tended to see more infections due to risky mobility patterns but fewer infections due to increased concern over COVID-19. States with more public health spending tended to have a strongly negative direct effect on the spread of infections, as we might expect given that public health practices can help spread information about the virus, but this association was partially offset by reduced concern over the pandemic and riskier travel patterns. Similarly, the number of health providers has a strong negative association with the virus that is entirely offset by reduced concern over the pandemic. Both of these covariates have as a consequence very small total effects, suggesting that the different pathways are obscuring the complex ways with which state health care resources affected the course of the pandemic.

The economy poll is another interesting case as on the whole it is strongly negatively associated with infections. As the percentage of people who believed the economy was in a good state rose, infections tended to decrease. Furthermore, this effect is primarily a direct effect, though there is some association with reduced infections via mobility. This result is theoretically interesting as trade-offs over the economy were often framed as a willingness to combat the epidemic versus the economic consequences of social distancing (Bonaccorsi et al. 2020b). The empirical analysis shows that this trade-off may exist and that fears over an economic downturn increased infections, though not by changing mobility patterns or concern over the severity of the disease. We might speculate that this association results from increased willingness to comply with economically costly social distancing behaviors when the economy is believed to be on sound footing, though without further analysis we cannot say for sure. 
What is clear is that the strongest time-varying factors present in the model concern individual behavior more than policies or state preparedness. Considering that the percentage of foreign residents (i.e., exposure to international travel) and per capita income were determined long before COVID-19 arrived, the most important manipulable factors are those involving beliefs, such as in the strength of the economy and the relative threat of COVID-19, along with personal behaviors like mask-wearing.

It is also interesting to note contrasting direct and indirect effects in panel A of Figure 10. The large effect from the COVID poll primarily comes from mobility data; people who are more concerned about COVID are less likely to frequent places where they could contract the disease. The mask poll is associated with repressing COVID through mobility, fear of COVID-19, and as a direct effect (presumably reduced spread through airways). The fact that all of these associations align suggests that the idea that masks would encourage risky behavior is in fact untrue (Abaluck et al. 2020). Finally, states with a larger proportion of smokers do tend to see more infections on a direct pathway, presumably by increasing people's risk to severe disease, but this effect is largely offset by increased fear in these states of the disease. This fascinating result shows how competing direct and indirect effects can mask an important empirical association we would expect given prior knowledge.

We next turn to the prominence of partisanship variables in explaining the spread of the disease, which we did not include in the previous figures as we interacted Trump vote share and within-state changes in approval polls in our model. Instead, we explore this interaction graphically in Figure 11. In this figure, the effect of Trump 2016 vote share is plotted conditional on the relative level of daily Trump approval polling on the $x$ axis. The effects are shown aggregated in panels A and B and disaggregated across mobility types in panel C. Panel A shows that in general, the effect of partisanship for Trump has both direct and indirect effects, with the direct effect highly conditional on the above/below polling average of approval for Trump in a given state (which has a maximum swing of about $+/-4 \mathrm{pp}$ ). When Trump approval rose, states with high Trump vote share witnessed fewer infections later on. These high conditional associations are likely due to the rally-around-the-flag effect in which Trump's approval rating spiked when the epidemic first appeared in March and April, leading to an association between high approval levels and low infection counts in conservative areas of the country.

However, it is important to note opposite effects through the mediated pathways. Panels B and C in Figure 11 shows that Trump vote share mediated through mobility and fear is strongly positive in terms of infection counts. While the effects are not as large as the direct effects, they are still substantial. Trump vote share's effect on COVID-19 mediated through these important channels shows that pro-Trump states tend to implement social-distancing behaviors at lower rates, as previous research has shown, with consequent 
relative increases in infections. Furthermore, these associations are relatively constant given Trump approval polls, although there is a more stronger association for combined Trump approval polling and Trump vote share in dampening fears over COVID-19; i.e., in states that voted the most for Trump, when Trump approval reached it highest point then fear of COVID-19 declined the most (and infections consequently increased).

On the whole, this finding points to very strong associations between partisanship and the spread of the COVID-19, comparable or greater than the demographic and socio-economic factors in the model. States with higher Trump vote shares have seen significantly fewer infections via unexplained pathways, but very importantly, this decrease did not come through reduced mobility nor increased concern over COVID-19. The direct relationship is likely an artifact of the pattern of the early spread of the virus. After all, it is well-known that the early states that were infected with COVID tended to vote against Trump, although partisanship is not why they were more vulnerable to COVID initially. We believe that pro-Trump states received fortuitous outcomes by happening to not be on major travel routes from early COVID-19 hot spots; rising Trump approval in these states occurred as pro-Trump residents believed their president's dismissal of the virus' threat. In other words, the unexplained direct effect justified the relative inattention to important behaviors that could prevent infection. Given the increase in COVID-19 infections in the last two months in heavily Republican states, it would seem that this tendency would lead pro-Trump states to suffer in the long run as behavior caught up with initial conditions.

Finally, we can also use estimates of cell phone mobility on COVID-19 to understand how NPIs have had mediated effects on the disease through increasing or decreasing mobility. Figure 12 shows the disaggregated mediation effects for two types of policies, restrictions on businesses and stay-at-home orders. The plots reveal how indirect mediation effects change substantially over time. Panel A shows that business restrictions had a powerful suppressive effect on workplace mobility and to a lesser extent retail establishments during the early part of the epidemic, though that association weakened over time. By contrast, panel B indicates that stay-at-home orders have had more durable effects on mobility that have suppressed the disease, particularly in retail establishments, workplaces and transit. Furthermore, these effects seem to be increasing rather than decreasing over time. On the other hand, stay-at-home orders seem to be increasing disease infections via increasing residential mobility, trade-offs that were noted in some early epidemiological modeling of COVID-19 (Seth Flaxman 2020).

To compare the effect of NPIs to that of partisanship we can consider a policy of a given length of implementation. Both business restrictions and stay-at-home orders reduced infections primarily via reducing retail mobility and workplace mobility, with stay-at-home orders with associations twice as high per day compared to business restrictions. For stay-at-home orders, a policy implementation of 30 days would equal 
A

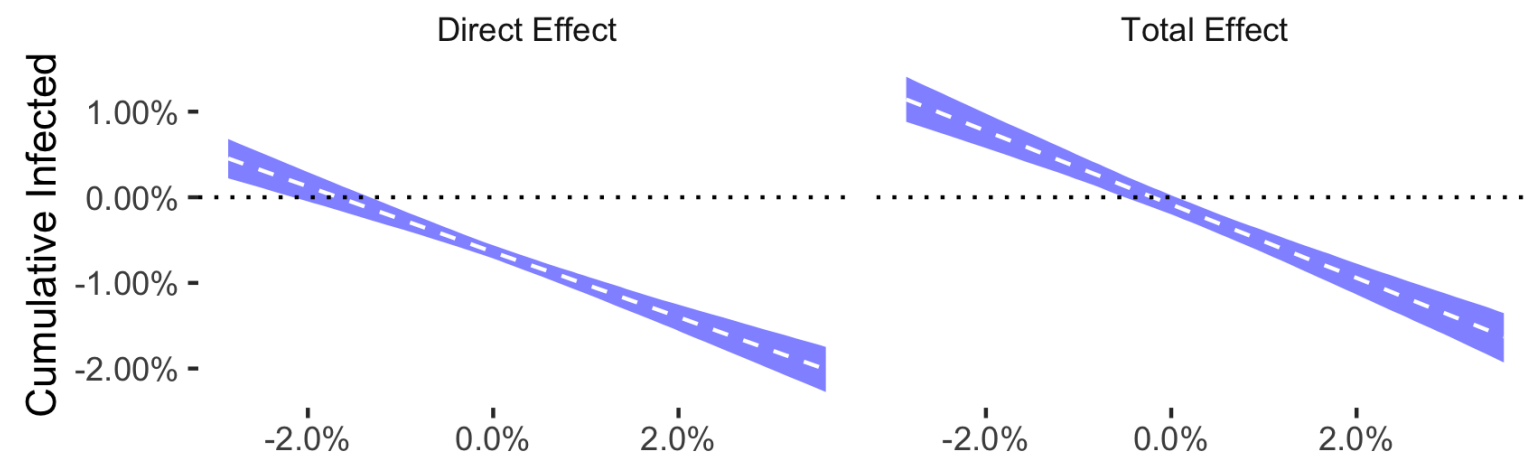

$\mathrm{B}$

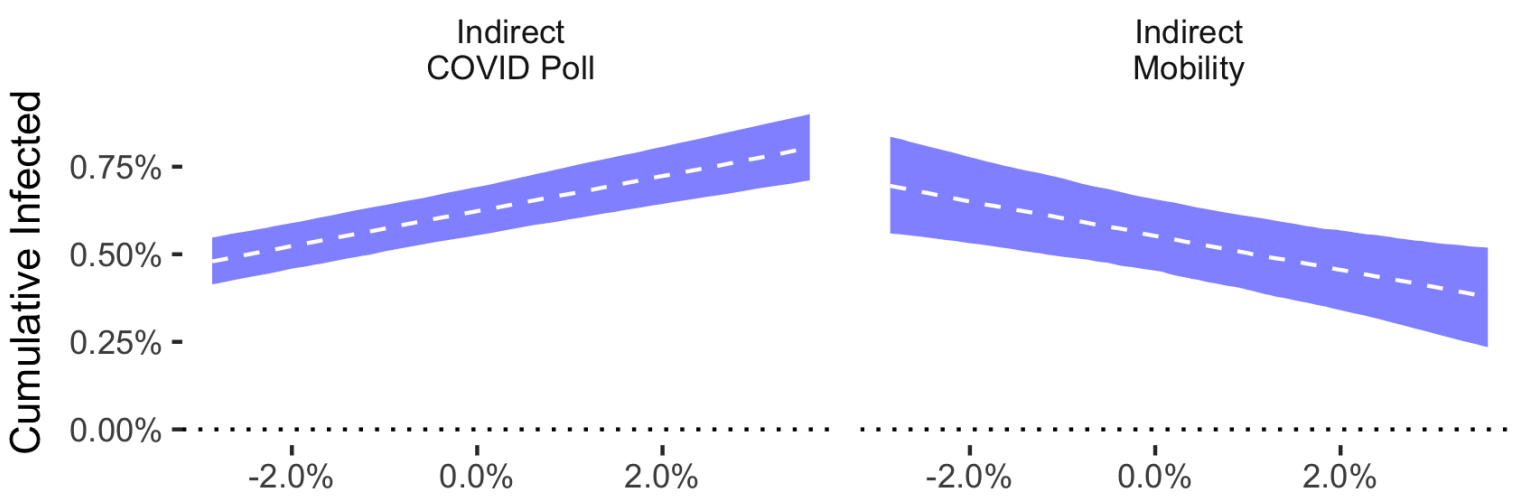

C

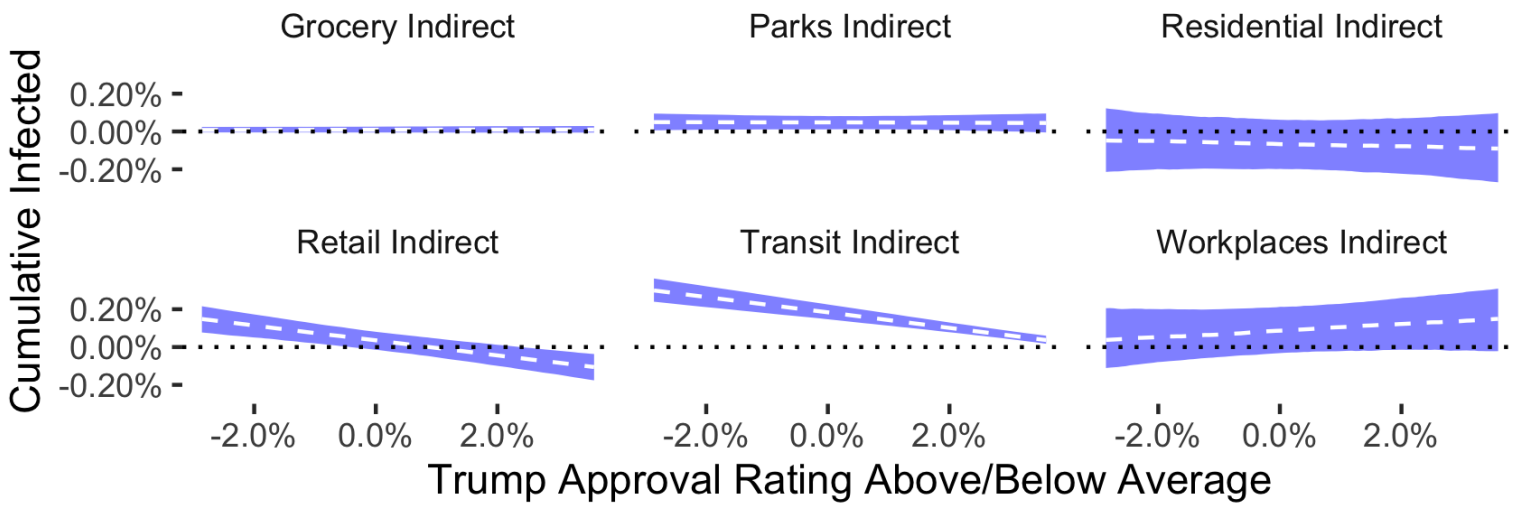

Plots show marginal effect of within-state increasing Trump approval rating conditional on a state's vote share for Trump in 2016.

Figure 11: Marginal Effects of Trump Vote Share in 2016 Conditional on State Approval Polls 

A Restrictions on Businesses $B$

Stay-At-Home Orders

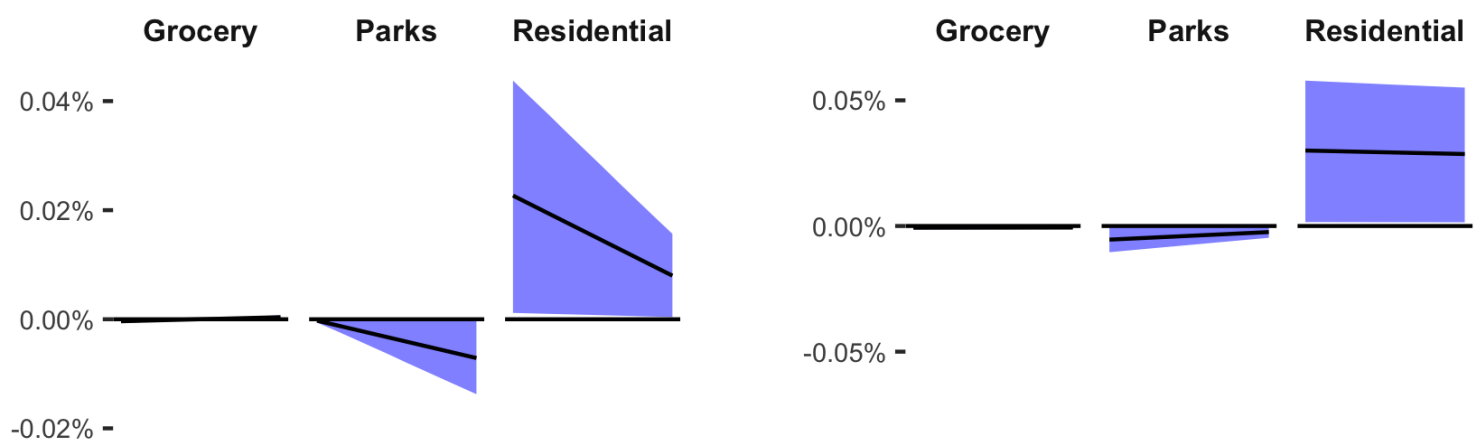

$-0.10 \%-$

Retail

Transit Workplaces

Retail

Transit

Workplaces

$0.04 \%-$

$0.05 \%=$

$0.02 \%-$
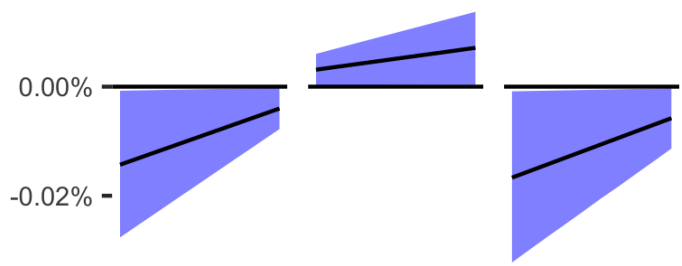

$\begin{array}{lccccccccccc}1 & 1 & 1 & 1 & 1 & 1 & 1 & 1 & 1 & 1 & 1 & \text { ' } \\ 0 & 50 & 100150 & 0 & 50 & 100 & 150 & 0 & 50 & 100 & 150\end{array}$ Time Since First Case (SDs)

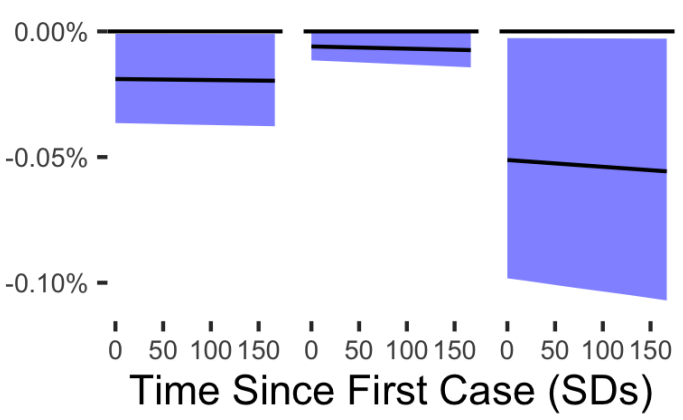

Results from mediation analysis using MCMC with Stan. Panel A shows indirect (via mobility data) and direct effects for business policy restrictions. Panel B shows direct and indirect (via mobility data) effects for stay-at-home policy restrictions.

Figure 12: Mediated Effects of Lockdowns on Google Mobility Data 
a reduction in infections via workplaces of approximately $1.5 \%$, while a business restriction policy would reduce infections via workplace mobility by approximately $0.6 \%$. As such, while stay-at-home orders did have large associations with subsequent spread of the virus, the effect magnitudes are comparable to the associations with Trump approval and Trump vote share. If we consider that Trump vote share increased infections by between $0.3 \%$ and $0.5 \%$ via mobility, we can further estimate that an additional stay-at-home order of at least 15 days duration would need to be imposed to compensate for the increased infections. This stay-at-home order would need to be even longer if we take into account offsetting increases in infections due to increased residential mobility.

\section{Conclusion}

Our results show that sociopolitical covariates like partisanship are equally as important predictors of the disease's spread as are NPIs designed to counter the pandemic. These results suggest that future research take into account these covariates even if they are not traditionally included in epidemiological studies. The politicization of the pandemic undermined measures to combat and led to reduced concern over the virus in the United States, with serious consequences for individuals' exposure to SARS-CoV-2. We find that politicization on the right end of the political spectrum has the strongest association with increased spread of COVID-19, although left-leaning political activity aimed at ending policy brutality via protests is also associated with increased spread, though at a lower scale.

The model employed in this article was devised to permit the statistical identification of suppression measures and social, political and economic factors on the spread of COVID-19. It is not intended to be a replacement or alternative to the disease forecasting literature. If anything, this modeling exercise shows why structural epidemiological models are so important: without them it is impossible to project the total number of infected people on a given day. This model's simplicity and ability to use empirical data are its main features, and the hope is that it can be used and extended by researchers looking at government policies and other tertiary factors on the spread of the disease. At the very least, the model provides realistic uncertainty intervals taking into account very real biases in the observed data.

\section{Bibliography}

Abaluck, Jason, Judith A. Chevalier, Nicholas A. Christakis, Howard Paul Forman, Edward H. Kaplan, Albert Ko, and Sten H. Vermund. 2020. "The Case for Universal Cloth Mask Adoption and Policies to 
Increase Supply of Medical Masks for Health Workers." SSRN.

Abouk, Rahi, and Babak Heydari. 2021. "The Immediate Effect of COVID-19 Policies on Social-Distancing Behavior in the United States." Public Health Reports, January, 0033354920976575. https://doi.org/10. $1177 / 0033354920976575$.

Alcott, Hunt, Levi Boxell, Jacob Conway, Matthew Gentzkow, Michael Thaler, and David Yang. 2020. "Polarization and Public Health: Partisan Differences in Social Distancing During the Coronavirus Pandemic." Journal of Public Economics.

Alesina, Alberto, and Howard Rosenthal. 1995. Partisan Politics, Divided Government and the Economy. Cambridge University Press.

Allcott, Hunt, Levi Boxell, Jacob C. Conway, Billy A. Ferguson, Matthew Gentzkow, and Benny Goldman. 2020. "What Explains Temporal and Geographic Variation in the Early US Coronavirus Pandemic?" https://doi.org/10.3386/w27965.

"America's Health Rankings 2019 Report." 2019. United Health Foundation. https://www. americashealthrankings.org/learn/reports/2019-annual-report.

Andersen, Martin. 2020. "Early Evidence on Social Distancing in Response to COVID-19 in the United States." SSRN.

Bonaccorsi, Giovanni, Francesco Pierri, Matteo Cinelli, Andrea Flori, Alessandro Galeazzi, Francesco Porcelli, Ana Lucia Schmidt, et al. 2020a. "Economic and Social Consequences of Human Mobility Restrictions Under COVID-19." Proceedings of the National Academy of Sciences 117 (27): 15530-35. https://doi.org/10.1073/pnas.2007658117.

- et al. 2020b. "Economic and Social Consequences of Human Mobility Restrictions Under COVID19." Proceedings of the National Academy of Sciences 117 (27): 15530-35. https://doi.org/10.1073/pnas. 2007658117.

Brauner, Jan M., Sören Mindermann, Mrinank Sharma, David Johnston, John Salvatier, Tomáš Gavenčiak, Anna B. Stephenson, et al. 2020. "Inferring the Effectiveness of Government Interventions Against COVID-19." Science, December. https://doi.org/10.1126/science.abd9338.

Brzezinski, Adam, Guido Deiana, Valentin Kecht, and David Van Dijcke. 2020. "The COVID-19 Pandemic: Government Versus Community Action Across the United States." CEPR Press, no. 7: 115-47.

Carleton, Tamma, and Kyle C. Meng. 2020. "Causal Empirical Estimates Suggest COVID-19 Transmission Rates Are Highly Seasonal." Working Paper. https://t.co/69vR0LUGsT?amp=1. 
Carpenter, Bob, Andrew Gelman, Matthew D. Hoffman, Daniel Lee, Ben Goodrich, Michael Betancourt, Marcus Brubaker, Jiqiang Guo, Peter Li, and Allen Riddell. 2017. "Stan: A Probabilistic Programming Language." Journal of Statistical Software 76 (1).

Cheng, Cindy, Joan Barcelo, Allison Spencer Hartnett, Robert Kubinec, and Luca Messerschmidt. 2020. "COVID-19 Government Response Event Dataset (CoronaNet v.1.0)." Nature Human Behavior. https://doi.org/https://doi.org/10.1038/s41562-020-0909-7.

Courtemanche, Charles, Joseph Garuccio, Anh Le, Joshua Pinkston, and Aaron Yelowitz. 2020. "Strong Social Distancing Measures in the United States Reduced the COVID-19 Growth Rate." Health Affairs 39 (7): 1237-46. https://doi.org/10.1377/hlthaff.2020.00608.

Dave, Dhaval M., Andrew I. Friedson, Kyutaro Matsuzawa, and Joseph J. Sabia. 2020. "When Do Shelterin-Place Orders Fight COVID-19 Best? Policy Heterogeneity Across States and Adoption Time." https: //doi.org/10.3386/w27091.

Dave, Dhaval M., Andrew I. Friedson, Kyutaro Matsuzawa, Joseph J. Sabia, and Samuel Safford. 2020. "Black Lives Matter Protests, Social Distancing, and COVID-19." NBER.

Dudel, Christian, Tim Riffe, Enrique Acosta, Alyson A. van Raalte, and Mikko Myrskyla. 2020. "Monitoring Trends and Differences in COVID-19 Case Fatality Rates Using Decomposition Methods: Contributions of Age Structure and Age-Specific Fatality." Working Paper. https://doi.org/10.31235/osf.io/j4a3d.

Fan, Ying, A. Yesim Orhun, and Dana Turjeman. 2020. "Heterogeneous Actions, Beliefs, Constraints and Risk Tolerance During the COVID-19 Pandemic." NBER.

Ferrari, Silvia, and Francisco Cribari-Neto. 2004. "Beta Regression for Modelling Rates and Proportions." Journal of Applied Statistics 31 (7): 799-815.

Gadarian, Shana Kushner, Sara Wallace Goodman, and Thomas B. Pepinsky. 2020. "Partisanship, Health Behavior and Policy Attitudes in the Early Stages of the COVID-19 Pandemic." SSRN.

Gao, Song, Jinmeng Rao, Yuhao Kang, and Yunlei Liang andJake Kruse. 2020. "Mapping County-Level Mobility Pattern Changes in the United States in Response to COVID-19." SIGSPATIAL Special 12 (1): $16-26$.

Gao, Song, Jinmeng Rao, Yuhao Kang, and Yunlei Liang andJake Kruse. 2020. "Mapping County-Level Mobility Pattern Changes in the United States in Response to COVID-19." SIGSPATIAL Special 12 (1): $16-26$. 
Grinsztajn, Léo, Elizaveta Semenova, Charles C. Margossian, and Julien Riou. 2021. "Bayesian Workflow for Disease Transmission Modeling in Stan." arXiv:2006.02985 [q-Bio, Stat], February. http://arxiv.org/ abs/2006.02985.

Grossman, Guy, Soojong Kim, Jonah M. Rexer, and Harsha Thirumurthy. 2020. "Political Partisanship Influences Behavioral Responses to Governors' Recommendations for COVID-19 Prevention in the United States." Proceedings of the National Academy of Sciences. https://doi.org/10.1073/pnas.2007835117.

Grossman, Matt, and David J. Hopkins. 2016. Assymetric Politics: Ideological Republicans and Group Interest Democrats. Oxford University Press.

Gu, Youyang. 2020. Covid19-Projections.com. https://covid19-projections.com/about/\#about-the-model.

Haug, Nils, Lukas Geyrhofer, Alessandro Londei, Elma Dervic, Amélie Desvars-Larrive, Vittorio Loreto, Beate Pinior, Stefan Thurner, and Peter Klimek. 2020. "Ranking the Effectiveness of Worldwide COVID19 Government Interventions." Nature Human Behaviour 4 (12): 1303-12. https://doi.org/10.1038/ s41562-020-01009-0.

Havers, C.; Lim, F. P.; Reed, and I Krapiunaya. 2020. "Seroprevalence of Antibodies to SARS-CoV-2 in 10 Sites in the United States." JAMA Internal Medicine.

Horowitz, Juliana Menasce, Anna Brown, and Kiana Cox. 2019. "Race in America 2019." Pew Forum.

Iyengar, Shanto, and Sean J. Westwood. 2015. "Fear and Loathing Across Party Lines: New Evidence on Group Polarization." American Journal of Political Science 59 (3): 690-707.

Jose Lourenco, Mahan Ghafari, Robert Paton. 2020. "Fundamental Principles of Epidemic Spread Highlight the Immediate Need for Large-Scale Serological Surveys to Assess the Stage of the SARS-CoV-2 Epidemic." medRxiv. https://doi.org/https://doi.org/10.1101/2020.03.24.20042291.

Larremore, Daniel B., Bailey K. Fosdick, Kate M. Bubar, Sam Zhang, Stephen M. Kissler, C. Jessica E. Metcalf, Caroline O. Buckee, and Yonatan H. Grad. 2020. "Estimating SARS-CoV-2 Seroprevalence and Epidemiological Parameters with Uncertainty from Serological Surveys." medRxiv, June, 2020.04.15.20067066. https://doi.org/10.1101/2020.04.15.20067066.

Neil M Ferguson, Gemma Nedjati-Gilani, Daniel Laydon. 2020. "Impact of Non-Pharmaceutical Interventions (NPIs) to Reduce Covid19 Mortality and Healthcare Demand." Imperial College of London Working Paper. https://www.imperial.ac.uk/media/imperial-college/medicine/sph/ide/gidafellowships/Imperial-College-COVID19-NPI-modelling-16-03-2020.pdf. 
Painter, Marcus, and Tian Qiu. 2020. "Political Beliefs Affect Compliance with COVID-19 Social Distancing Orders." SSRN.

Peak, Corey M., Rebecca Kahn, Yonatan H. Grad, Lauren M. Childs, Ruoran Li, Marc Lipsitch, and Caroline O. Buckee. 2020. "Modeling the Comparative Impact of Individual Quarantine Vs. Active Monitoring of Contacts for the Mitigation of COVID-19." medRxiv. https://doi.org/https://doi.org/10.1101/2020. 03.05.20031088.

Perkins, T. Alex, Sean M. Cavany, Sean M. Moore, Rachel J. Oidtman, Anita Lerch, and Marya Poterek. 2020. "Estimating Unobserved SARS-CoV-2 Infections in the United States." Working Paper. http: //perkinslab.weebly.com/uploads/2/5/6/2/25629832/perkins_etal_sarscov2.pdf.

Poole, Keith T., and Howard L. Rosenthal. 2007. Ideology $\&$ Congress. Transaction Publishers.

Poole, Keith, and Howard L. Rosenthal. 1997. Congress: A Political-Economic History of Roll Call Voting.

Riou, Julien, Anthony Hauser, Michel J. Counotte, and Christian L. Althaus. 2020. "Adjusted Age-Specific Case Fatality Ratio During the COVID-19 Epidemic in Hubei, China, January and February 2020.” medRxiv. https://doi.org/https://doi.org/10.1101/2020.03.04.20031104.

Robert Verity, Ilaria Dorigatti, Lucy C Okell. 2020. "Estimates of the Severity of COVID-19 Disease." medRxiv. https://doi.org/https://doi.org/10.1101/2020.03.09.20033357.

Ruiyun Li, Bin Chen, Sen Pei. 2020. "Substantial Undocumented Infection Facilitates the Rapid Dissemination of Novel Coronavirus (SARS-CoV2)." Science. https://doi.org/10.1126/science.abb3221.

Sajadi, Mohammad M., Parham Habibzadeh, Augustin Vintzileos, Shervin Shokouhi, Fernando MirallesWilhelm, and Anthony Amoroso. 2020. "Temperature, Humidity and Latitude Analysis to Predict Potential Spread and Seasonality for COVID-19.” SSRN. https://papers.ssrn.com/sol3/papers.cfm?abstract_ $\mathrm{id}=3550308$.

Sánchez-Romero, Miguel, Vanessa di Lego, Alexia Prskawetz, and Bernardo L. Queiroz. 2021. "An Indirect Method to Monitor the Fraction of People Ever Infected with COVID-19: An Application to the United States." PLOS ONE 16 (1): e0245845. https://doi.org/10.1371/journal.pone.0245845.

Seth Flaxman, Axel Gandy, Swapnil Mishra. 2020. "Estimating the Number of Infections and the Impact of Non-Pharmaceutical Interventions on COVID-19 in 11 European Countries." Working Paper. https: //www.imperial.ac.uk/mrc-global-infectious-disease-analysis/covid-19/report-13-europe-npi-impact/.

Sharma, Mrinank, Sören Mindermann, Jan Markus Brauner, Gavin Leech, Anna B. Stephenson, Tomáš Gavenčiak, Jan Kulveit, Yee Whye Teh, Leonid Chindelevitch, and Yarin Gal. 2020a. "How Robust Are 
the Estimated Effects of Nonpharmaceutical Interventions Against COVID-19?" arXiv:2007.13454 [Cs, q-Bio, Stat], December. http://arxiv.org/abs/2007.13454.

—. 2020b. "How Robust Are the Estimated Effects of Nonpharmaceutical Interventions Against COVID-19?" arXiv:200\%.13454 [Cs, q-Bio, Stat], December. http://arxiv.org/abs/2007.13454.

Stekhoven, Daniel J., and Peter Bühlmann. 2012. "MissForest-Non-Parametric Missing Value Imputation for Mixed-Type Data." Bioinformatics 28 (1): 112-18.

Tasnim, Samia, Md Mahbub Hossain, and Hoimonty Mazumder. 2020. "Impact of Rumors or Misinformation on Coronavirus Disease (COVID-19) in Social Media." SocArchiv. https://doi.org/10.31235/osf.io/uf3zn.

Winship, Christopher, and Robert D. Mare. 1983. "Structural Equations and Path Analysis for Discrete Data." The American Journal of Sociology 89 (1): 54-110.

Yuan, Ying, and David P. MacKinnon. 2009. "Bayesian Mediation Analysis." Psychological Methods 14 (December): 301-22. https://doi.org/10.1037/a0016972. 